\title{
NEW CLASS MAGNETORHEOLOGICAL ELASTOMERS BASED ON WASTE TIRE RUBBER AND PROPERTIES CHARACTERIZATION
}

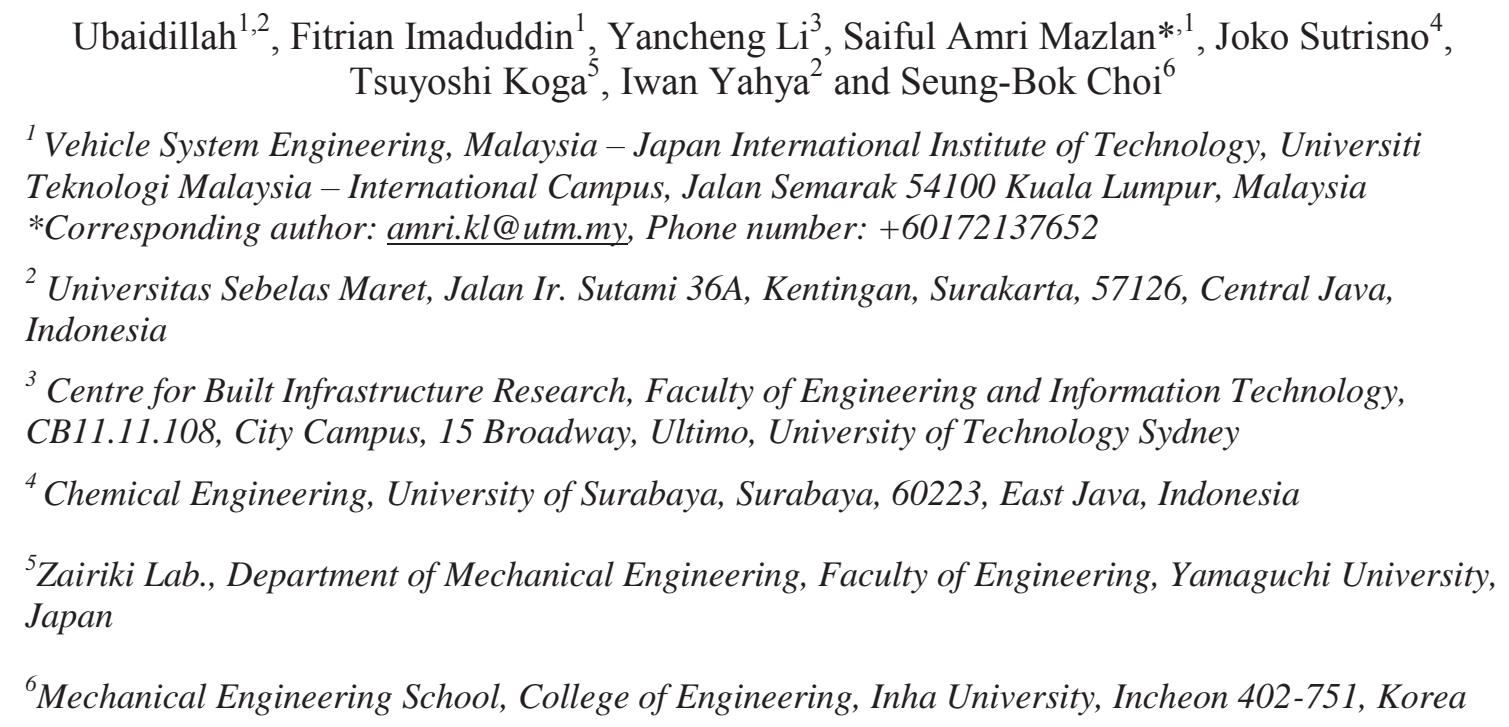




\section{1. Introduction}

2 For many years, researchers have been actively seeking a means of rubber reclamation due to

3 the growing amount of rubber waste especially from scrap tires [1]. Scrap tire rubber is 4 considered to be harmful waste due to the poor decomposition resulting in serious 5 deterioration of the water, land, and air qualities. Many strategies have been developed for 6 tire rubber recycling such as land filling, material, and energy recovery, and reusing for other 7 functional items, such as virgin rubber filler, concrete and asphalt mixture, as well as 8 composite materials [2]. However, since the common rubber recycling process only places

9 the scrap rubber as a supplementary material, the value of the scrap rubber is very low and 10 often unattractive for recycling effort. The value of the scrap tire rubbers would be higher if it 11 can be involved not just in the synthesis of conventional rubber mixture but also in the 12 fabrication process of a more valuable material such as the smart composites.

13 Magnetorheological elastomer (MRE) as one of the smart composite materials have taken a 14 prominent position in semi-active damping devices such as automotive suspension, rotating 15 machine mounting, and seismic damper, due to their responsiveness, and tunability properties 16 to the magnetic fields $[3,4]$. This smart composite consists of the matrix, additive, and 17 magnetizable particles. The matrix usually comes from saturated elastomers (silicone), 18 unsaturated rubber (natural and synthetic rubber), and thermoset or thermoplastic elastomers 19 (polyurethane). The magnetizable particles are commonly carbonyl iron particles (CIP) or 20 other iron oxides. However, to the authors' knowledge, as yet, in the last two decades 21 researchers have only disclosed about the fabrication process of MREs using the virgin 22 rubber matrix while the discussion of waste rubber processing as the MREs matrix has not 23 been reported yet.

24 Semi-active devices, which incorporate MRE as the controllable material, are expected to be 25 functionalized in multi-directional deformation. Therefore, the viscoelastic properties of all 26 modes should be explored to establish the MR effect relationship in different types of 27 loading, i.e. both shear and normal. Recently, although investigation of the shear mode has 28 been widely conducted, the compression method of MRE has received less attention [5,6]. 29 The stress-strain response in static compression load provides a Young's modulus that is 30 dependent on the magnetic field. The static compression test was first conducted by Farshad 31 and Benine [7]. In this research, a silicone rubber based MRE was statically loaded until a 32 certain strain amplitude revealing the Young's modulus. Other similar methods were reported 33 for different types of MRE, such as urethane [8]; different shaped MREs [9]; and urethane 
1 foam based MRE [10,11]. Other static compression effects of MRE have been studied in 2 respect of various attractive properties, e.g. electrical conductivity, which is dependent on the 3 magnetic field $[12,13]$. Meanwhile, the effect on the compression rate and pre-compression 4 normal force have been reported by Liao et al. [14,15]. Although the static compression test 5 has shown the remarkable properties of the MRE, it is not sufficient for exploring their 6 rheological properties.

7 The dynamic compression test has been widely used for investigating the viscoelastic 8 properties of elastomers. However, the dynamic compression of MREs has received less 9 attention compared to static compression [5,6,16,17]. Kallio et al [5] tested isotropic and 10 anisotropic silicone-based MREs to observe the stiffness under different magnetic field 11 strength and found that the maximum adjustment of achievable spring constant was $10 \%$. Li 12 and Sun [6] investigated the viscoelastic properties of isotropic and anisotropic silicone-based 13 MRE using compression and shear method in swept frequency and strain. In 2009, Koo et al. 14 [16] performed the dynamic compression test to develop a neural network based 15 phenomenological model of isotropic silicone based MRE based on force-displacement 16 relationship. Meanwhile, similar testing method was used by Song et al. [17] to investigate 17 viscoelastic properties of the MRE influenced by the filler's shape factor i.e. spherical and 18 nanowire ferrous powder. Since the dynamic compression in these four studies has similarity 19 in the selected matrix i.e. silicon rubber, it can be assumed that there are no major differences 20 in the fabrication technique of the MREs. However, these works did not consider the 21 development of magnetic field apparatus for rheological testing; which is essential for 22 estimating the value of flux density across the MRE during rheological test.

23 The main technical contributions of this work are summarized as follows; 1) fabrication of a 24 new MRE using ground tire rubber and 2) material characterizations via step-by-step 25 examination. In this work, the fully vulcanized scrap tire rubber is introduced as the new 26 matrix for MRE. The successful processing of the scrap tire rubber is undertaken using the 27 high-temperature high-pressure (HTHP) sintering method which converts inert scrap tires 28 into recycled rubber [18]. The HTHP is a waste rubber reclamation process that is conducted 29 by applying simultaneous high temperature (between $200{ }^{\circ} \mathrm{C}$ to $240{ }^{\circ} \mathrm{C}$ ) and high pressure 30 compaction (above $20 \mathrm{MPa}$ ) on the ground tire rubber in a mold for particular duration. The 31 process does not involve the twin screw extruder that operates through shearing the waste 32 rubber at temperature of $\pm 180{ }^{\circ} \mathrm{C}$. The fabrication method of the MRE using this technique is 33 considered to be a new approach since virgin elastomer based MREs are processed in 
1 different ways. After making several samples, physical characterization of the MREs was

2 undertaken to explore their microstructure and magnetic properties, together with thermal

3 analysis. A detailed explanation of the development of the magnetic field source was also

4 undertaken to predict the flux distribution within the test specimens. Validation of the

5 electromagnetic device was also performed by comparing the simulation results with the

6 measurements recorded in the experiment. Finally, the rheological properties of the waste

7 rubber based MRE were investigated focusing on the magnetorheological effect that is an

8 inherent property of smart materials.

\section{2. Materials and Preparation}

10 2.1. Raw Materials

11 The matrix materials originated from scrap tire rubber, while the magnetizable particles were 12 derived from ferrite block materials. The scrap tires purchased from a company ( PT;

13 Bengawan Sumber Baru, Indonesia) were in powder form and undefined size. The source of 14 scrap tire was purely from the waste tire rubber and no contaminant from other kinds of 15 rubber waste was present. The scrap tire rubber was separated from all metals and fabrics.

16 The rubber powder of larger size was then shredded in the laboratory using a 60-mesh rubber 17 granulator to achieve the uniformity of powder size. The chemical properties of the crumb 18 rubber given by the manufacturer were as follows [18]: acetone extract $7 \%$, ash content $5.4 \%$, 19 carbon black $32.9 \%$, and hydrocarbon rubber $54.6 \%$. The additives used in this study were 20 latex solution 15\% (10 phr), sulfur ( $2 \mathrm{phr}$ ), zinc oxide (5 phr), and stearic acid (1.5 phr).

21 The magnetizable powder was synthesized using a high energy ball mill. The ferrite block 22 obtained from CV Oriental Electronic, Indonesia, was crushed using a hydraulic press until 23 the powders passed 60-mesh size. The screened powder was then milled using a custom made 24 high energy ball mill with an angular velocity of $500 \mathrm{rpm}$. The mass ratio of powder to ball 25 mill was 1:10. In this work, 50 g powder was ground using a $500 \mathrm{~g}$ hardened steel ball in 50 $26 \mathrm{ml}$ ethanol. The ground powder was then dried at $70^{\circ} \mathrm{C}$ for an hour. The ground powder was 27 examined to determine its physic properties, and the results were identified as magnetite 28 particle $\left(\mathrm{Fe}_{3} \mathrm{O}_{4}\right)$ based on their diffraction and transmittance patterns.

29 2.2. Fabrication

30 The fabrication of the MRE specimens consisted of two major steps namely mixing and 31 reclaiming. Since the expected type of MRE was isotropic, electromagnetic treatment was 32 absence during reclamation process. In this work, four variations of MRE samples were 
1 synthesized i.e. 10, 20, 30, and $40 \mathrm{wt} \%$ of filler fractions. A laboratory-made mixer was 2 utilized for mixing crumb rubber, additive, and magnetite powder at $250 \mathrm{rpm}$. The 3 homogenous mixture was quickly achieved since all the raw materials were in powder form. 4 The mixing was firstly conducted for $100 \mathrm{phr}$ of crumb rubber with $2 \mathrm{phr}$ sulfur, $5 \mathrm{phr}$ zinc 5 oxide, and $1.5 \mathrm{phr}$ stearic acids using a mechanical mixer. The first mixing is undertaken for $6 \quad 15-30$ minutes. When mixing the matrix materials, 10 phr latex solution $15 \%$ was added and 7 mixed for 15 minutes. Since the total mass of molded materials was set to 10 grams each, the 8 weight comparisons of rubber mixture and magnetite powder were 9:1, 8:2, 7:3, and 6:4 for $910 \mathrm{wt} \%, 20 \mathrm{wt} \%, 30 \mathrm{wt} \%$, and $40 \mathrm{wt} \%$ of MREs, respectively. By taking the appropriate 10 mass of rubber mixture and magnetite powder, the matrix and fillers were then mixed 11 homogeneously. The final step was placing the mixture in a high-temperature high-pressure 12 (HTHP) mold having a diameter of $30 \mathrm{~mm}$.

13 The molding was then mounted in the HTHP device, as shown in Figure 1. The HTHP 14 equipment was constructed from several parts; hydraulic unit, heater controller and heater 15 element. The sintering of the MRE specimens was performed by first applying a hydraulic 16 pressure of $25 \mathrm{MPa}$ to the molding followed by heating at $200^{\circ} \mathrm{C}$. The maximum heating rate 17 achieved by the controller was about $10^{\circ} \mathrm{C} / \mathrm{min}$. During sample preparation, the initial 18 temperature was about $25^{\circ} \mathrm{C}$ to $27^{\circ} \mathrm{C}$ (room temperature) transiently increasing until $200^{\circ} \mathrm{C}$ 19 for 17-20 minutes. After steady state temperature, the controller continuously supplied 20 electric current to the heater elements based on the temperature set point. The HTHP process Figure 1 also depicts the fabricated MRE samples in coin shape. The size of the specimen especially thickness (measured with displacement sensor) depends on the composition of the mixture since the sintered mixtures had the same mass. The higher magnetite content produced a lower sample thickness. The density of specimens was measured using density meter for further identification of the equivalent volume fraction. The densities and filler 27 fraction also reveal the linear relationship. The assessment of volume fraction was derived 28 using Eq. 1 [19];

$$
\varnothing=\frac{d_{M R E S}-d_{W}}{d_{M P}-d_{W}}
$$

Eq. 1

where $d_{M R E S}$ is the density of MREs; $d_{W}$ is the density of pure reclaimed rubber and $d_{M P}$ is

31 the density of magnetite powder. The base density for pure reclaimed rubber and magnetite 32 powder were $1.107 \mathrm{~g} / \mathrm{cm}^{3}$ and $5.27 \mathrm{~g} / \mathrm{cm}^{3}$, respectively. 


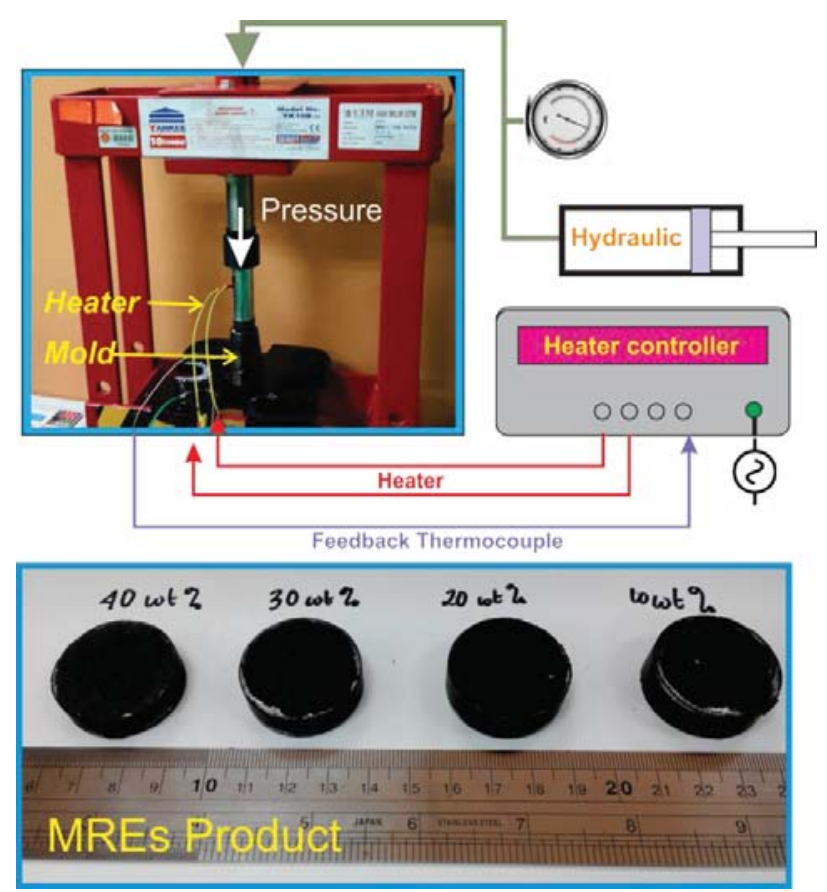

Figure 1. HTHP fabrication device and the resulting MRE specimens

\section{3. Experimental and Apparatus}

\section{3.1. Microstructural Observation}

5 Microstructural observation was undertaken to provide the information concerning the 6 magnetite shape, size, and dispersion within the matrix. Scanning electron microscopy 7 (SEM), FEI-Inspect was utilized to capture the magnetite powders distribution within the 8 matrix. The device was operated at an accelerating voltage of $10 \mathrm{kV}$ (magnetite powder 9 examination) and $5 \mathrm{kV}$ (microstructure of the MRE). In SEM observation, the MRE samples 10 were shattered to expose their interior. The observed surfaces were coated with a thin layer of 11 gold. The particle size distribution (PSD) of the magnetite was measured using Shimadzu

12 Particle Size Analyzer 2300, Kyoto, Japan.

\section{3.2. Magnetic Measurement}

14 The magnetic characterization is carried out using Lake Shore vibrating sample 15 magnetometry (VSM). The tests were conducted before and after MRE fabrication. The 16 initial evaluation was addressed to characterize the laboratory-prepared magnetite powders. 17 This test is beneficial for determining the relative magnetic permeability of the pure 18 magnetite particles. Meanwhile, the post-fabrication test was undertaken to evaluate the 19 magnetic properties of the MRE samples. The experimental curves were measured at ambient 20 temperature after applying a maximum field intensity of 1 Tesla. The magnetic properties of 
1 the laboratory-prepared magnetite as well as MRE samples were measured at room

2 temperature from -1 to 1 Tesla.

3 3.3. Thermal Characterization

4 The importance of thermal analysis for the MRE is mainly associated with the stability and

5 degradation of the elastomers. Differential scanning calorimetry (DSC) was utilized to reveal

6 the thermal properties of the waste based MRE. Examination is necessary since the issue for

7 reclaimed rubber is its thermal property and stability during operation in extreme condition.

8 The DSC test covered pure reclaimed waste rubber and the MRE in various weight fractions

9 for assessment of thermal glass transition temperature. The Shimadzu DSC-60A utilized in

10 this study was adaptable to the step-scan method. The method was conducted in combination

11 with power compensation. The technique dissociated the reversible and irreversible reactions

12 by employing a sequence of short interval cooling from ambient temperature to $-100{ }^{\circ} \mathrm{C}$ using

13 liquid nitrogen, which was then heated to $0{ }^{\circ} \mathrm{C}$. The convection cooling rate was set 10

$14{ }^{\circ} \mathrm{C} / \mathrm{min}$.

15 3.4. Electromagnetic Device

16 It is commonly known that the compression behavior of elastomeric materials is strongly

17 influenced by the interface condition between the pressure plate and the specimen [20].

18 According to this situation, the rubber compression test is divided into three states - bonded,

19 free friction, and single fixed end. The non-linear dynamics of compressed rubber exhibit a

20 different behavior according to the compression state in which the natural behavior of the

21 compressed elastomer can be revealed by implementing the bonded state. The specimen is

22 bonded permanently to the compression plate at the surface tips. In this situation, the

23 compressed rubber shows the highest achievable modulus or stiffness. The dynamic

24 compression of the MREs performed in this study employed the bonded state.

25 The dynamic compression test of the MREs must be conducted using a permanent magnet

26 [16] or electromagnetic device [5]. The flux density of the permanent magnet can be

27 controlled by adjusting the distance between two adjacent magnets. Although utilizing a

28 permanent magnet seems simple, the span of the flux density capability is narrower than that

29 for an electromagnetic. The other disadvantage is that the flux density drops as the distance

30 between the permanent magnets increases [16]. This research utilized an electromagnetic

31 device with functions for generating high flux density as well as compression. Figure 2

32 depicts the cross sectional view and the fabricated electromagnetic unit. 
2 Figure 2. Electromagnetic unit (1.upper jaw, 2.upper coil, 3.cover, 4.lower coil, 5.lower jaw)

3 It is important to note that the electromagnet utilized in the rheological test should be capable

4 of producing a higher flux density within the test area than the magnetization saturation $\left(M_{S}\right)$

5 of the MRE samples. In addition, the magnetic flux generated within the test area of the

6 electromagnetic device must be easily monitored by the operator. Moreover, the coils should

7 be in direct contact with the free air for heat discharge when the electric current is applied.

8 Based on the mentioned requirements, the design of the electromagnetic device was

9 developed as depicted in Figure 2. The electromagnetic device mainly consists of two coil-

10 bobbin sets, steel cover, and center jaws. One of the center jaws (lower jaw) is fixed to the

11 load cell while the other moves with the hydraulic actuator. The movement of the upper jaw

12 should be free from the static parts with no friction, as friction will affect the reading of the

13 load sensor.

14 The electromagnetic parts, such as the number of wire turns, were first determined through

15 magnetic circuit analysis. The present electromagnetic design was capable of generating more

16 than $500 \mathrm{mT}$ in the test area without MREs with a gap thickness of $12 \mathrm{~mm}$. According to the

17 provided bobbin space, each coil was calculated as having 1250 turns, a wire diameter of 1

$18 \mathrm{~mm}$, wire resistance of $12.6 \mathrm{ohms}$, and a maximum current of $5 \mathrm{~A}$. The jaws and cover

19 materials were AISI 4140; for the magnetic properties for the software input refer to Shahreza

20 et al. [21]. 
$1 \quad 3.5$. Dynamic test method

2 The simplified system of the compression test is illustrated in Figure 3. The compression 3 mode of MREs is identical to the lumped spring elements consisting of the cylindrical MRE 4 subjected to a cyclic compression force for off-state and on-state (external magnetic field 5 application). A Shimadzu ServoPulser L Series, Shimadzu, Kyoto, Japan, was used to 6 perform the compression test having maximum force ranges of $\pm 20 \mathrm{kN}$. The fatigue dynamic 7 machine has a functional excitation frequency range of 0.1 up to $50 \mathrm{~Hz}$, depending on the 8 excitation amplitude (10 to $0.9 \mathrm{~mm}$ ). The Shimadzu Servo Controller 4830 was equipped 9 with user interface software that could produce the force and displacement data in the time 10 domain to obtain force-displacement curves. The stress-strain relationship was also 11 determined by the software according to the dimension of the MRE.

12 The dynamic compression tests were performed at various excitation frequencies of $1,3,5,7$, 1310,13 and $16 \mathrm{~Hz}$ with a constant strain amplitude of $10 \%$. Meanwhile, various swept strain 14 amplitudes were used - 1, 3, 5, 7 and 10\% - under $1 \mathrm{~Hz}$ excitation frequency. The strain 15 amplitude was controlled according to the cyclic equation of $\varepsilon=\varepsilon_{a} \sin (\omega t)$, where $\varepsilon_{a}$ is 16 strain amplitude and $\omega=2 \pi f$ is the excitation frequency in $\mathrm{rad} / \mathrm{s}$. In practice, the dynamic 17 machine regulates the excitation frequency $f$ in Hz. The MRE specimen was subjected to the 18 harmonic loading that fluctuates about the mean level (half of strain amplitude). During the 19 compression test, the current from the DC power supply was regulated from 0 to $5 \mathrm{~A}$. The temperature of the electromagnetic core, which was in direct contact with the sample, was monitored as the current drained to the coil. The temperature was kept below $60^{\circ} \mathrm{C}$ to maintain the condition of the specimen.

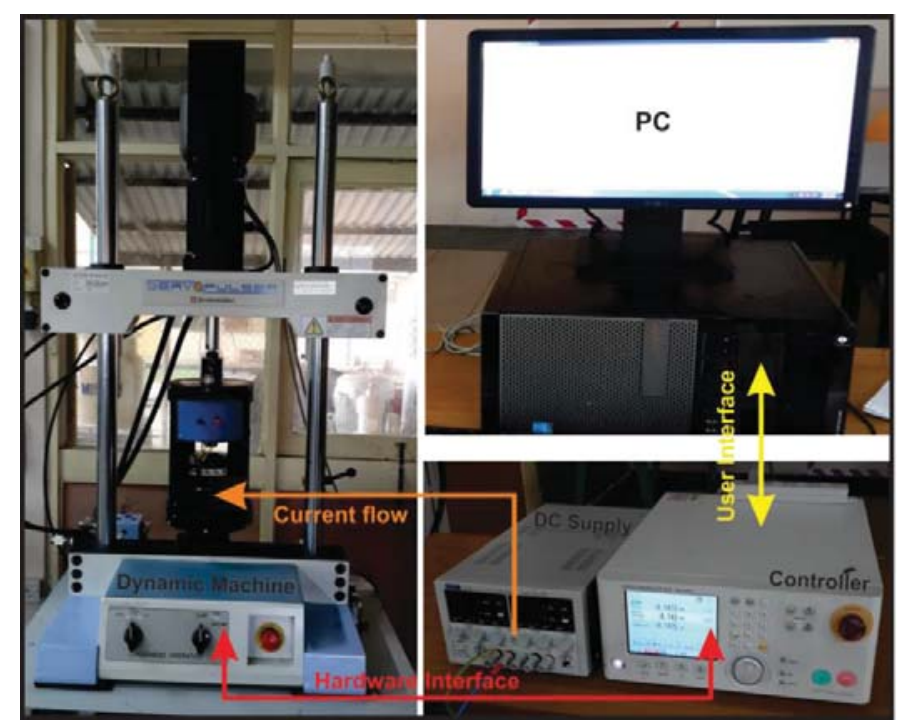


2 The rheological properties of various elastomers have been studied by applying cyclic stress 3 or strain. This technique applies to all linear viscoelastic materials. When the MRE sample is 4 subjected to oscillating strain, a stress also develops an indirect response in the time domain 5 as $\sigma=\sigma_{a} \sin (\omega t+\delta)$. The symbol $\delta$ represents the phase shift between the stress and strain.

6 The phase shift indicates the specific characteristic of a viscoelastic material. The associated 7 quantity to determine the property is $\tan \delta$ or loss tangent/loss tangent. The key property of 8 the MRE can be calculated using the simple relationship given in Eq. 2:

9

$$
\left|E^{*}(\omega)\right|=\frac{\sigma_{a}}{\varepsilon_{a}}
$$

Eq. 2

where, $E^{*}$ is the complex modulus. The $E^{*}$ and $\tan \delta$ are key viscoelastic characteristics of 11 the MRE [22]. The complex modulus consists of real and imaginary parts expressed as 12 follows (Eq. 3):

$$
E^{*}=E^{\prime}+i E^{\prime \prime}
$$

Eq. 3

where, $E^{\prime}$ is the storage modulus and $E^{\prime \prime}$ is the loss modulus. These two values are suitable for explaining the viscoelastic material properties of the MREs. The quantity of storage modulus and loss modulus can be determined from the relationship in the following 17 equations.

$$
E^{\prime}=E^{*} \cos \delta
$$

Eq. 4

$$
E^{\prime \prime}=E^{*} \sin \delta
$$

Eq. 5

20 The relative magneto-rheological effect (MR effect) can be further determined by

$$
\text { MR Effect }=\frac{\left(E_{\text {on-state }}-E_{\text {off-state }}\right)}{E_{\text {off }- \text { state }}} \times 100 \%
$$

Eq. 6

\section{Results and Discussions}

In this section, all experimental results are presented; the discussions cover the physical properties of the MRE including microstructure, magnetic properties, and thermal properties. The electromagnetic device magnetostatic simulation and experimental validation are also explained as well as the viscoelasticity in compression mode. Some parameters are varied including filler concentration, magnetic flux, excitation frequency and strain amplitude. The estuary of viscoelastic properties is to identify mechanical characteristics, such as storage modulus, loss modulus, and loss tangent of the waste rubber based MRE by applying different magnetic field intensities. 
4.1. Microstructure Analysis

2 The morphology and size distribution of magnetite powder is shown in Figure 4. The

3 particles have narrow distribution with a size of 0.5-25 microns according to the scale and 4 irregular shape. Based on the particle size analysis test, the particle size distribution is 5 measured as $\mathrm{d} 10=6.517 \mu \mathrm{m}, \mathrm{d} 50=16.919 \mu \mathrm{m}$, and $\mathrm{d} 90=44.142 \mu \mathrm{m}$. Meanwhile, the

6 photograph of the MRE interior containing magnetite powders with the weight fraction of 40

$7 \quad \mathrm{wt} \%$ is also portrayed in Figure 4. A well-dispersed magnetite powders was found within the

8 MRE matrix. It can be seen that the magnetite powder penetrates deep in the matrix without

9 any voids. The absence of voids is caused by the high pressure applied during HTHP process.

10 The phenomenon also implies that there is no oxidation occurred within the reclaimed rubber.

11 The average size of magnetite is finer than the tire rubber powders. Therefore, the mechanical 12 mixing allows the magnetite penetrates easily to the tire rubber powders. At early stage, the 13 homogenous mixture of magnetite and other raw materials could be easily observed visually.

14 During the HTHP process, the magnetite could not easily move since it was restricted by the 15 high pressure applied. As a result, the good dispersion was obtained as shown in Figure 4. 16 Well-dispersed particle is primary requirement of the consistent performance of the isotropic 17 MRE. The responsiveness of the MRE to the external magnetic flux is also influenced by the 18 dispersion of magnetic particles [19,23,24]. A good distribution will ensure the magnetic flux 19 flows uniformly through the MREs. The dispersion of particles can be provided in direct 20 evidence by scanning electron microscopy. Similar phenomena were also found in the lower 21 weight fraction of magnetite powder.
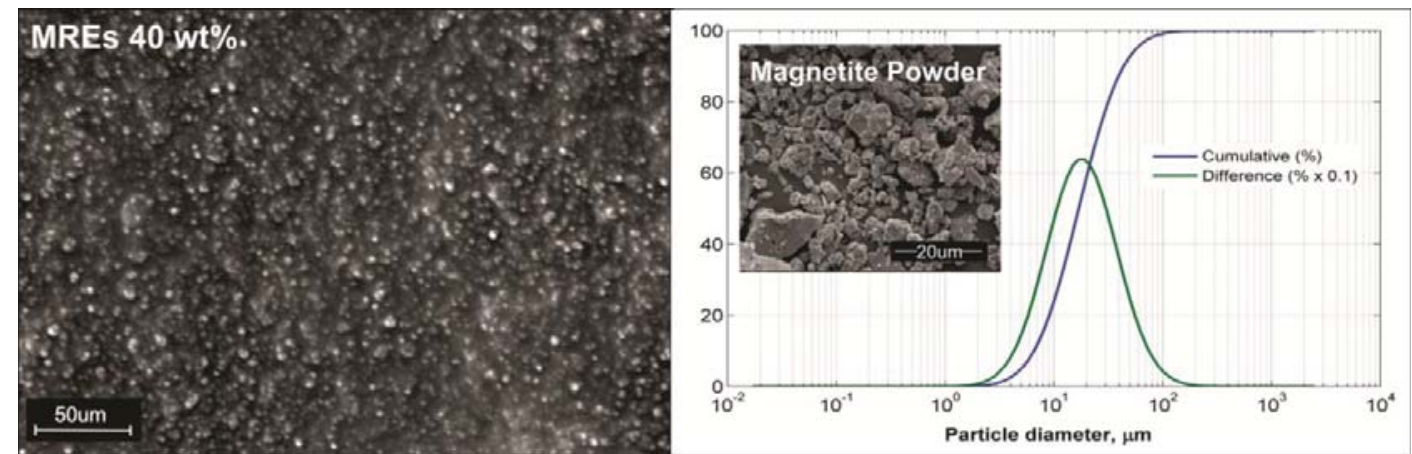

23 Figure 4. Microstructural properties of the sliced MRE as well as particles shape and size

24 4.2. Magnetic Properties

25 The hysteresis curves of the MRE in various weight fraction of the magnetite are depicted in 26 Figure 5. The figure displays the hysteresis loops for different MRE samples i.e. 10, 20, 30 27 and $40 \mathrm{wt} \%$. Table 1 shows the corresponding magnetic properties of the MREs including 
1 their retentivity, magnetization, and coercivity. The thin loops of the hysteresis curves show

2 that the MREs belong to the magnetically soft property at the ambient temperature. The

3 coercivity $\left(\mathrm{H}_{\mathrm{c}}\right)$ of all samples proves the soft magnetic properties in which the values are

4 lower than 90 Oe $(7161.972 \mathrm{~A} / \mathrm{m})$ [25]. The quantity of the coercivity is reduced with the

5 alteration of the filler content. Meanwhile, the magnetization $\left(M_{s}\right)$ and retentivity $\left(M_{r}\right)$ act on

6 the reverse values. The measured properties are parallel to the studies on magnetite-

7 thermoplastic natural rubber nanocomposites reported by Kong et al. [26] and polymeric

8 composite based barium ferrite reported by Ahmed et al. [27]. It could be inferred from the

9 higher values of the coercivity, that it would be harder to demagnetize the MRE at a lower

10 filler content. The increase in magnetization and retentivity is also in-line with the quantity of

11 magnetic susceptibility.

12 Table 1. Magnetic properties of the MREs with the increment of filler fraction

\begin{tabular}{cccc}
\hline Sample & $\begin{array}{c}\text { Retentivity, Mr } \\
\text { (emu/g) }\end{array}$ & $\begin{array}{c}\text { Magnetization, Ms } \\
\text { (emu/g) }\end{array}$ & $\begin{array}{c}\text { Coercivity, Hc } \\
\text { (A/m) }\end{array}$ \\
\hline $10 \mathrm{wt} \%$ MREs & 0.221 & 7.216 & 3435.54 \\
$20 \mathrm{wt} \%$ MREs & 0.873 & 14.185 & 3355.30 \\
$30 \mathrm{wt} \%$ MREs & 1.326 & 20.723 & 3339.09 \\
$40 \mathrm{wt} \%$ MREs & 1.859 & 27.709 & 3265.65 \\
Pure Magnetite & 4.7 & 85.775 & 2799.69 \\
\hline
\end{tabular}

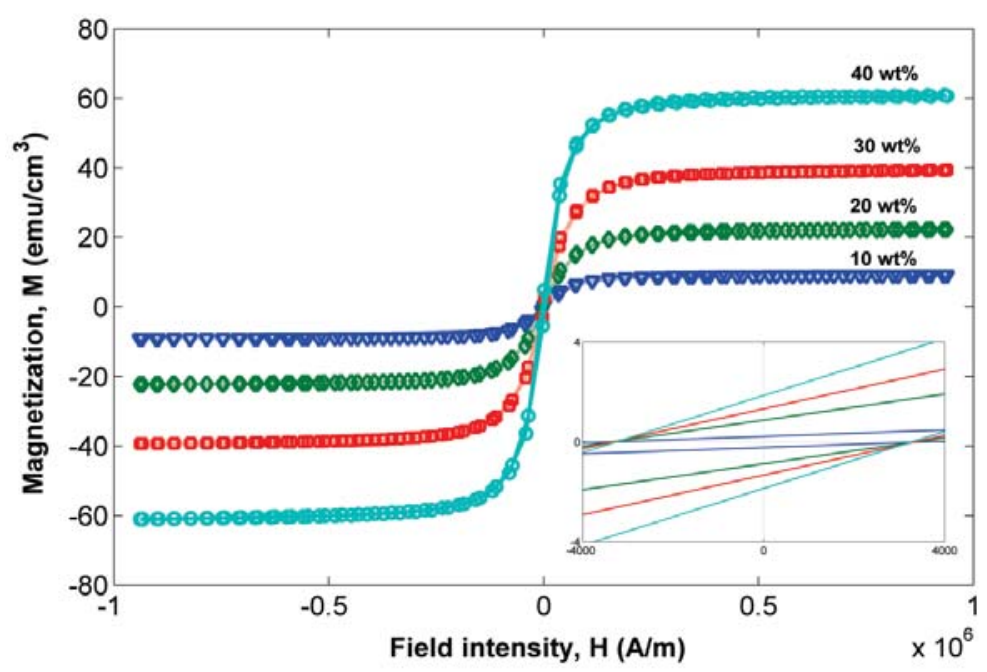

Figure 5. Magnetization curves of various MREs

\subsection{Thermal Analysis}

17 The influence of the filler particle on the thermal properties of reclaimed rubber was investigated. Differential scanning calorimetry (DSC) analysis was conducted to examine the 
1 characteristic temperatures of phase transformation in this reclaimed rubber matrix. Figure 6 2 shows the DSC curves of the MRE samples for various weight fractions of magnetite 3 compared with the pure reclaimed scrap tire rubber. The reclaimed rubber was synthesized 4 using the same procedure as that of the MRE samples. All the plotted curves exhibit the same 5 pattern in which the compatibility of the matrix and magnetizable particles are exhibited in 6 the single enthalpy exothermic phase. The phase transition (glassy state to rubbery state) 7 shows an endothermic reaction at an interval of -65 to $-55^{\circ} \mathrm{C}$. The thermal glass transition 8 temperatures $\left(T_{g}\right)$ were obtained using analytical software, and the $T_{g}$ value ranges were $9-0.6 \pm 0.5{ }^{\circ} \mathrm{C}$ for all measured samples, independent of the filler fraction. The calculated $T_{g}$ 10 also confirms that the value is equivalent to the thermal properties of vulcanized virgin 11 styrene butadiene rubber-natural rubber (SBR-NR), studied by Kim et al. [28]. The insertion 12 of magnetite powder does not have a substantial effect on the thermal glass transition 13 temperature. There is no evidence of the influence of the magnetite powder fraction on the 14 rubber curing during the reclamation process. Magnetite powder dispersed within the rubber 15 matrix has a better thermal conductivity than the matrix itself. Moreover, in composite 16 materials, the thermal conductivity is strongly influenced by the fraction of thermally 17 conductive materials. The better thermal conductivity and higher weight fraction of the 18 magnetizable particles is also indicated by the up-shift in the curves for each sample. The 19 higher fraction of particles can cause an increment in the ability of heat flow across the 20 samples. Consequently, the larger weight fraction of the MREs commonly has the better 21 thermal conductivity.

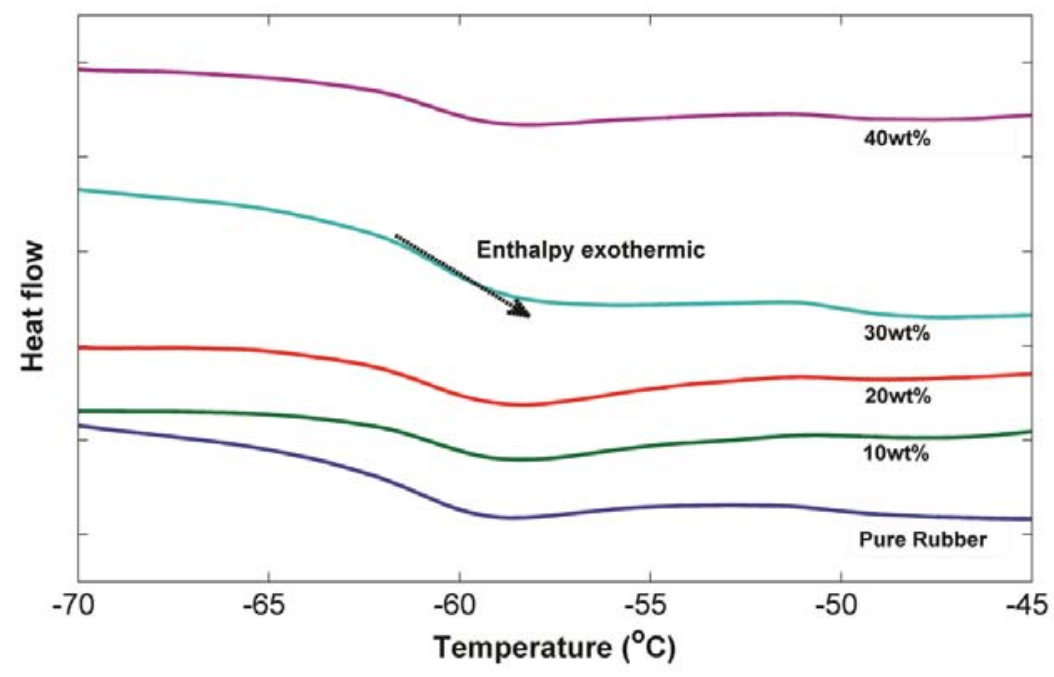

Figure 6. DSC curve of pure rubber and MRE samples 


\section{4.4. Electromagnetic Validation}

2 The performance of the electromagnetic apparatus was evaluated through simulation and 3 experimental activities. Figure 7(a) shows the magnetic flux distribution within the 4 electromagnetic device obtained from magnetostatic simulation. The flux lines passing 5 through the steel jaws, cover, and air gaps with slight leakage outside the instrument form a 6 closed loop magnetic circuit. The flux lines are directed in a parallel direction to the 7 movement of the upper jaw because the current flows perpendicular to the jaw. The 8 concentration of magnetic flux along the jaws confirms the strength of flux density within the 9 chamber test. The color distribution in the air gap, as shown in the zoomed picture, indicates 10 the uniformity of the flux density, despite the flux fringing around the air gap. The 11 magnetostatic simulation plotted in Figure 7(a) resulted from a 1 A current applied in each 12 coil. The simulation is performed in various clearances of the test area and currents. The air 13 gap distances are set to be 4,8 and $12 \mathrm{~mm}$. Meanwhile, the currents are varied from 0 to $5 \mathrm{~A}$ 14 by an interval of $0.25 \mathrm{~A}$. The average flux at the center of the test is presented in flux density 15 as a function of the applied current. Figure 7(b) shows the increment of the flux density at 16 various distances and currents. The patterns show that the increase in gap causes a marked 17 decrease in the flux density. The maximum flux densities with a 5 A current are $0.83,1.14$, 18 and $1.77 \mathrm{~T}$ for 12,8 , and $4 \mathrm{~mm}$ clearances, respectively. The maximum achievable flux 19 density for $12 \mathrm{~mm}$ gap exceeds the design expectation. Justification of the magnetostatic 20 simulation results is conducted by comparing the simulation results with the measured flux 21 density across the test area after the prototype is developed. The magnetic flux was measured 22 using a Gaussmeter GM08, Hirst Magnetic Instrument, United Kingdom, using the same jaw 23 distance as the simulation. The current was regulated by a power supply (TTi CPX 4000, a 24 maximum current of $20 \mathrm{~A} \mathrm{DC}$ ) of 0 to 5 A. Figure 7(b) depicts the comparison between the 25 simulation and the measurement results. Since the differences between the quantities are still 26 acceptable, the simulation model can be considered to be valid, and, hence, can be used for 27 further reference. 


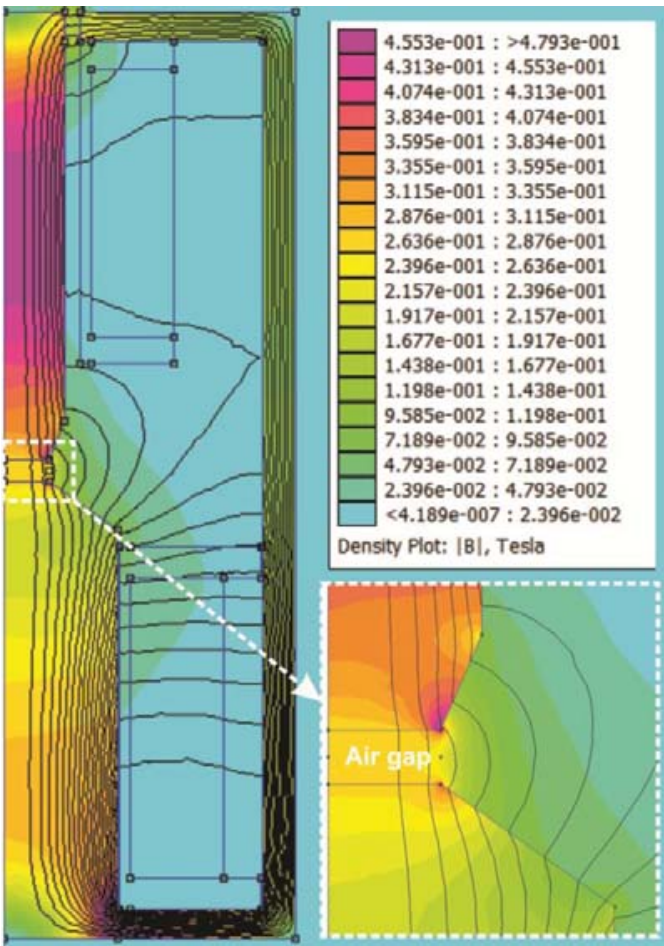

(a)

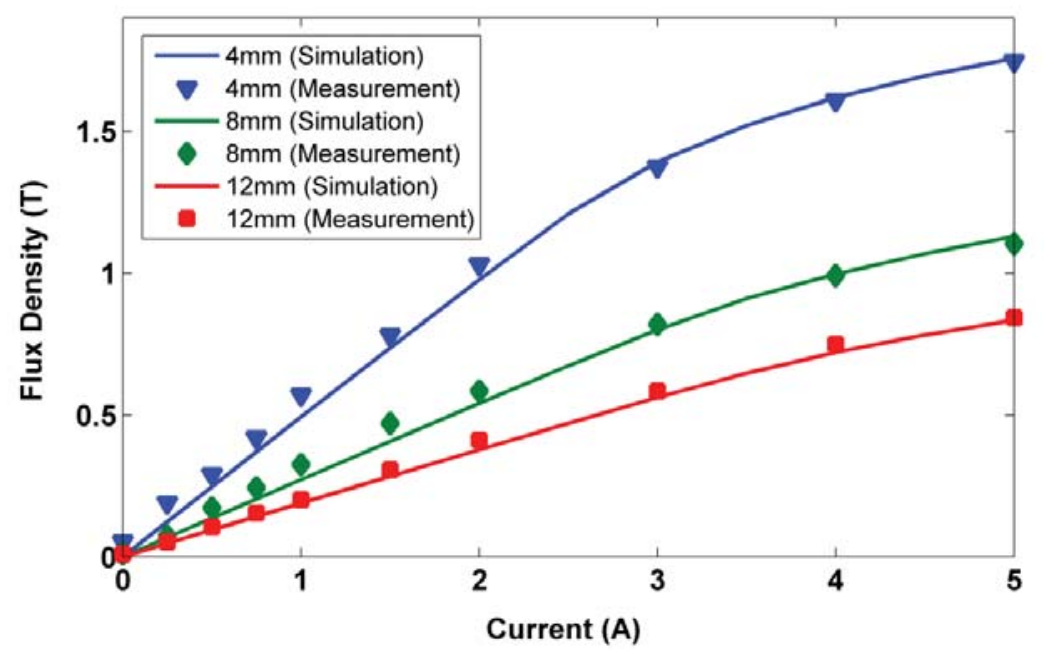

(b)

$5 \quad$ Figure 7. (a) FEMM simulation without MREs sample; (b) results in validation for various

gaps and currents

7 The MRE samples vary in thickness and have different magnetic properties. The previous

8 simulation is only valid for the condition without the MRE sample, which means the test area

9 has no sample. The following discussion is addressed on the magnetostatic simulation when

10 the MRE is placed in the test area. The importance of this simulation is mainly due to the

11 absence of a sensor, which can measure the flux density inside the materials. Based on the 
1 limitation, most of the previous works used a gaussmeter and placed it near to the MREs

2 being tested. The magnetic flux reading through the aforementioned procedure is less 3 accurate since the gaussmeter probe does not measure the exact flux density within the MRE.

4 Therefore, the proposed magnetostatic simulation helps the prediction of the flux density 5 passing through the MRE samples. Next, the air gap in the magneto-static model is then 6 replaced by the MRE including the magnetic properties and dimension of the simulation 7 setting parameters. The magnetic properties are derived from the measurement data. The 8 magnetic field distribution depicted in Figure 8(a) shows the simulation result for MRE 40 9 wt $\%$ (10.55 mm thickness) in a 1 A current. The figure portrays the higher density of the 10 magnetic flux across the MREs compared to the air gap, and also the uniformity of both the 11 lateral and axial direction. Since the jaws interconnected to the MREs have a good magnetic 12 permeability, the magnetic flux flows easier within the MRE. Figure 8(b) summarizes the 13 simulation results for all specimens. The input parameters are the variation in thickness 14 depending on the type of MRE and current applied to the coils. The current is varied from 0 15 to $5 \mathrm{~A}$. The flux density values are also logged from the average value across the sample. 16 From the figure, it can be inferred that the flux density escalates according to the increment in 17 the current applied as well as the augmentation of the particle weight fraction. The highest 18 flux density achieved by the MRE $10 \mathrm{wt} \%$ is about $1000 \mathrm{mT}$ at $5 \mathrm{~A}$ applied current. The 19 value is sufficient for magnetizing the MRE samples. Figure 8(b) can also be used as a 20 reference for equivalency between the electric current and flux density since the upcoming 21 viscoelastic behaviors are provided in the electric current domain.

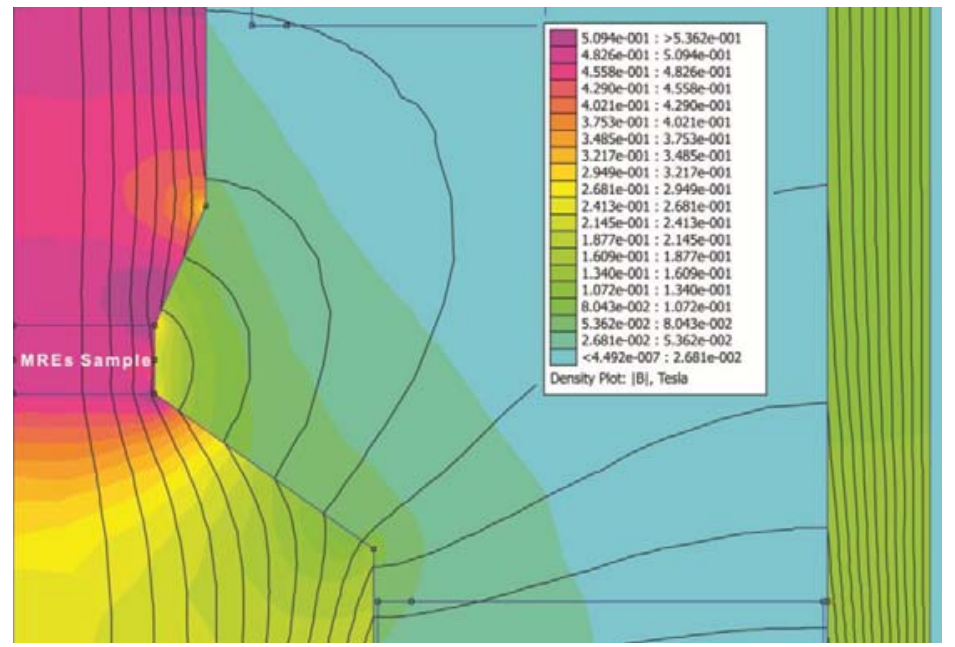




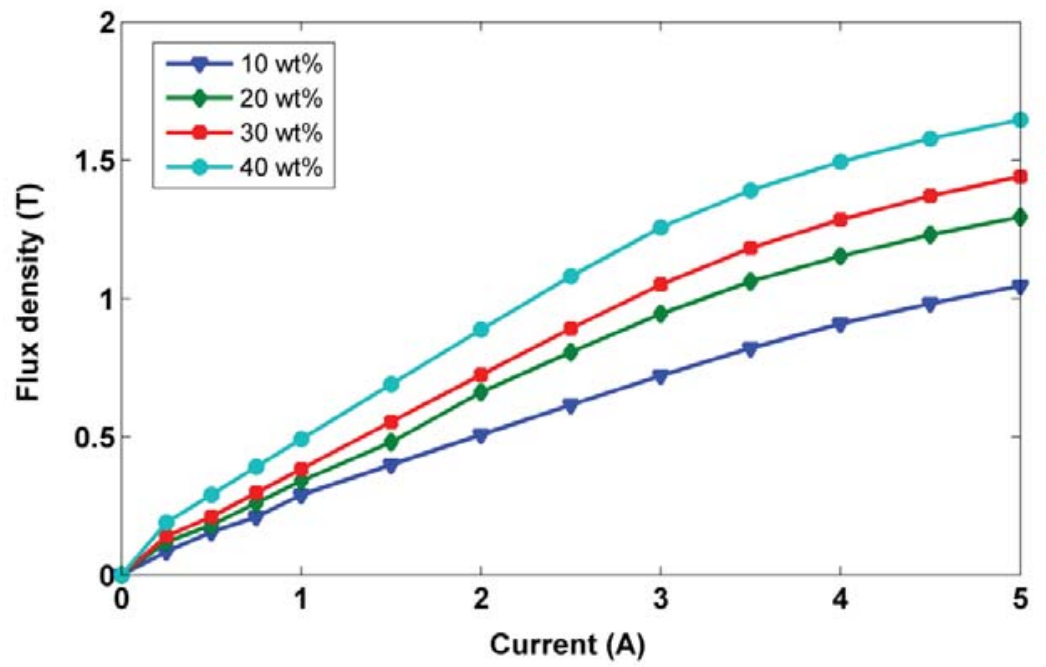

(b)

3 Figure 8. (a) Field distribution across the MRE samples; and (b) Quantity of flux density in various MRE types and applied current

\section{4.5. Viscoelastic Properties}

6 As mentioned earlier, the base matrix used in the MREs originated from scrap tire rubber. It

7 is commonly known that tire rubber has a high content of carbon black. The insertion of 8 carbon black in a virgin rubber during the former tire production strongly influences the 9 modulus characteristics. The analytical relationship between the modulus of virgin rubber and carbon black filled rubber has been extensively studied earlier. The theoretical frame of particle filled rubber has been established by Guth [29] based on the theory of viscosity. The modulus of ordinary particle filled rubber is stated as follows:

$13 \quad E^{*}=E_{i}\left(1+2.5 \phi+14.1 \phi^{2}\right)$

14 where, $E^{*}$ is the filled rubber modulus, $E_{i}$ is the initial modulus, and $\phi$ is the volume fraction 15 of filler. The equation is also applicable to the other constants, such as shear modulus $(G)$ and 16 stiffness modulus $(K)$; since, the $E, G$ and $K$ have a linear relationship. Figure 9 shows the modulus of pure reclaimed rubber and various types of MRE. The modulus of unfilled rubber is used as the initial modulus value $E_{i}$ for Guth's model prediction. The measured modulus of the MREs is then superimposed with the prediction result. The modulus of various weight fractions follows the prediction with acceptable deviation. The viscoelastic behavior of the

21 waste rubber based MREs is introduced in the form of the dynamic stress-strain relationship.

22 The stress-strain data are obtained in the time domain and further examined to determine the 23 other parameters. All the samples are tested and configured to monitor the stress-strain 
1 relation under the magnetic field. Due to the extensive data logged in various treatments, this

2 section only shows the stress-strain characteristics of a particular MRE.

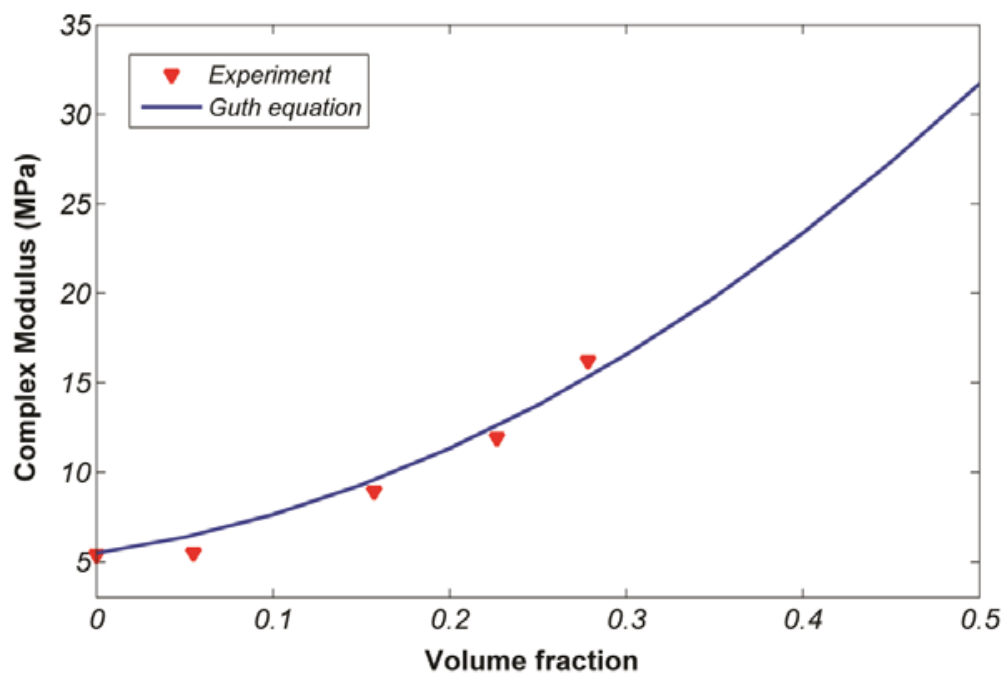

Figure 9. Off-state complex modulus of the MREs in various volume fractions

Figure 10(a) shows the stress-strain curves of MRE $40 \mathrm{wt} \%$ with various applied currents under cyclic loading. The testing is conducted with an excitation frequency of $1 \mathrm{~Hz}$ and $10 \%$ strain amplitude. The stress-strain curves were derived from the force-displacement data.

Therefore, the hysteresis trends must be identical. The MRE stiffness can be determined from the force-displacement signals. However, the primary objective of this study is to explore the field-dependent modulus. From the figure, it can be inferred that the near-elliptical loops change according to the current increment. The gradient of the major axis varies with the magnetic flux. This also confirms the alteration of the MRE stiffness. The areas covered by the loading and unloading curve also alter according to the increase in the current. This indicates that the damping capacity also varies according to the different magnetic fields. The maximum stress at $10 \%$ strain amplitude increases gradually as the current is applied from 0 to 3 A. However, the stress quantity changes slightly from 3 to $5 \mathrm{~A}$. This occurrence indicates that the stress increment does not increase when a higher magnetic flux is applied to the MRE. Figure 10(b) and (c), respectively, depict the hysteresis curve for various frequencies and strain amplitudes with a constant current. The different testing frequencies shown in the graph are performed under constant strain amplitude of $10 \%$ and 1 A current. The stress increases steadily with a relatively similar difference. There is no saturation of the stress increment caused by the higher excitation frequency. The continuous rise in peak frequency causes a change in the area of the near-elliptical loops. This fact physically means that the stiffness and damping properties are also altered. It is also observed that the damping 
1 capacity of the MRE can change the compression-relaxation response; namely, the phase lag.

2 Figure 10(c) presents the stress-strain curves for various strain amplitudes at a constant 3 frequency of $1 \mathrm{~Hz}$, and a constant current of $1 \mathrm{~A}$. When the strain amplitude is changed 4 sequentially, the little strain amplitude actuation provides a small stress value.

5

6

7

8

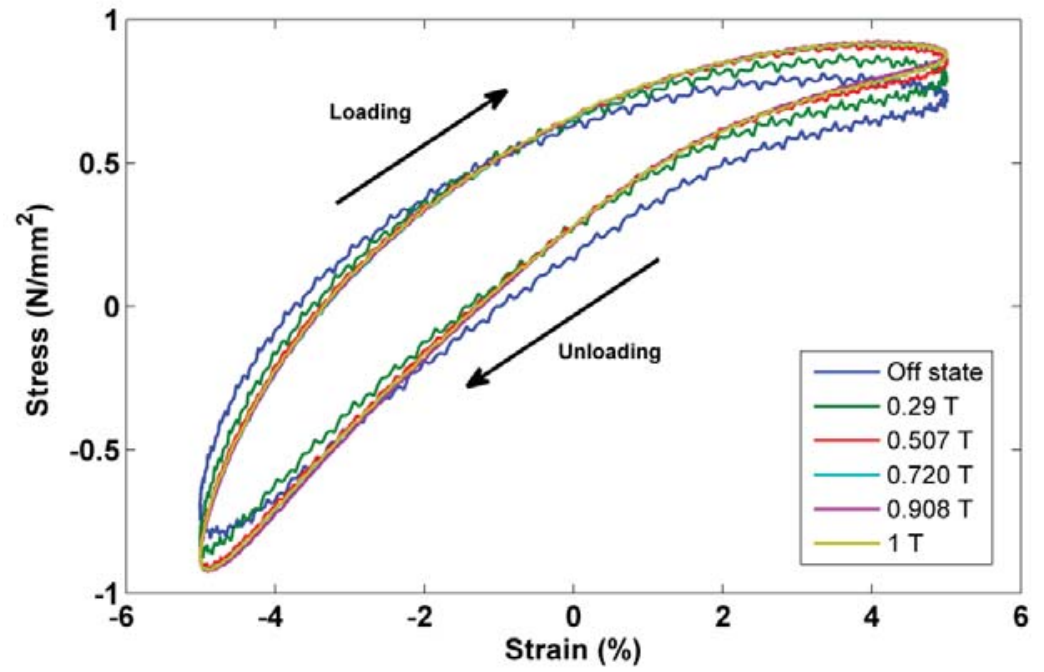

(a)

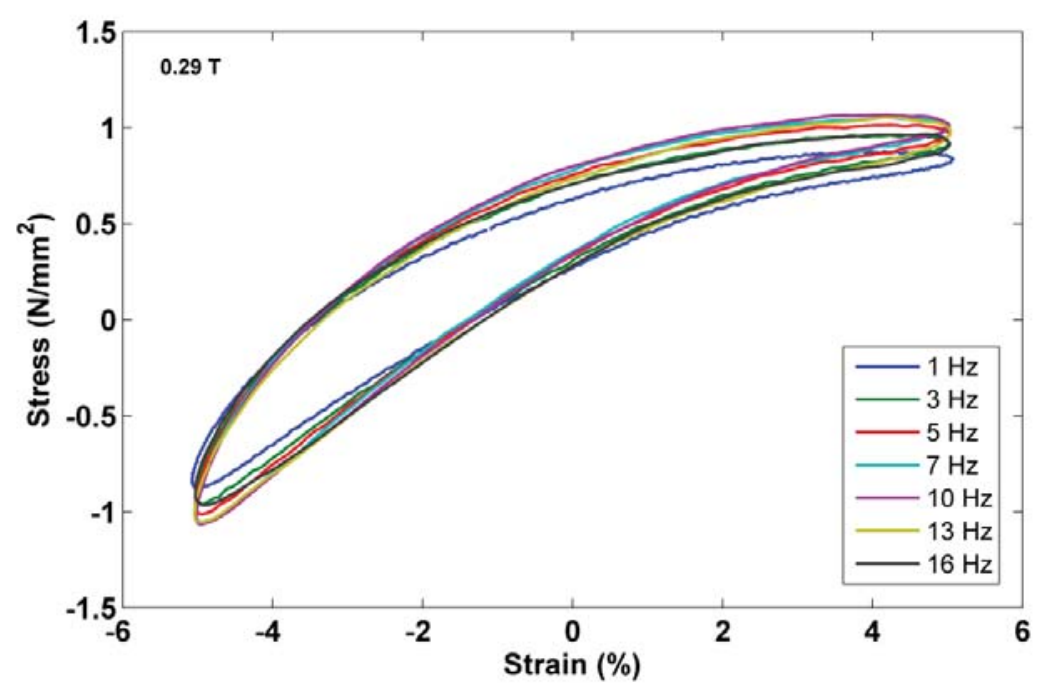

(b) 


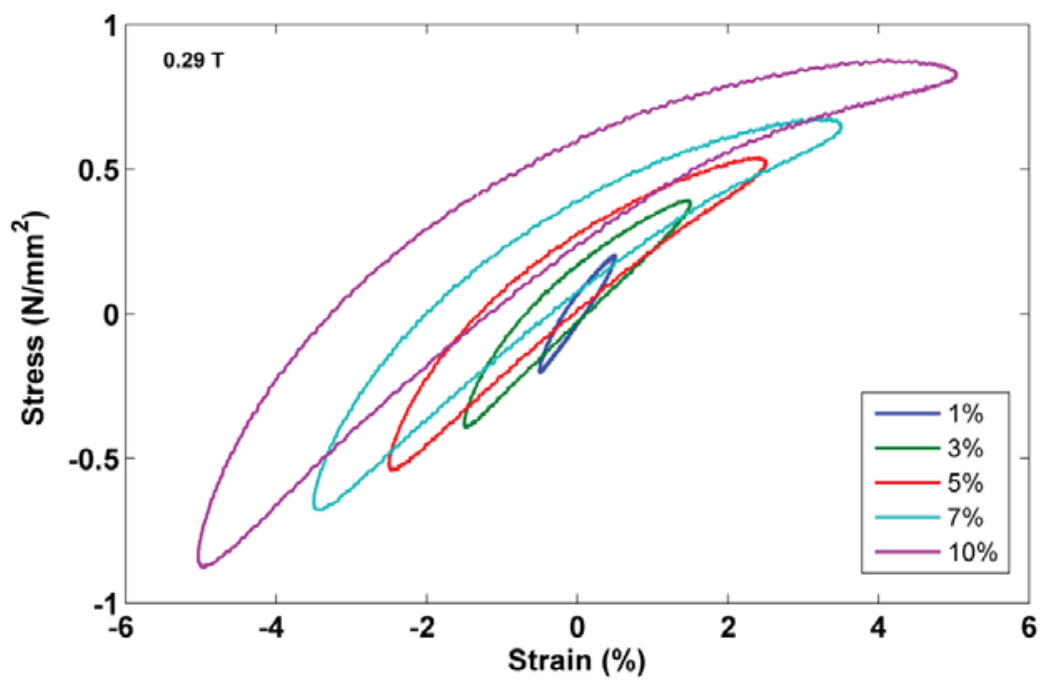

(c)

3 Figure 10. Hysteresis stress strain curves for $40 \mathrm{wt} \%$ MREs: (a) various currents, (b) various excitation frequencies, and (c) various excitation amplitudes

5 It is commonly known that the elastomer stress-strain curve in response to cyclic loading has 6 a symmetric elliptical shape. Therefore, the stress-strain curves for the lower amplitude are 7 covered by the stress-strain curves at a higher strain. The situation shown in Figure 10(c) is 8 rather different to that commonly found in the previous works [5,30]. The stress-strain 9 response for $1 \%$ amplitude seems to be symmetrical between the loading and unloading 10 states. However, the shape of the curve becomes asymmetrical with the higher excitation 11 strain. From, the phenomena it can be inferred that the higher compression strain in the axial 12 direction causes longer displacement in the internal particles. However, the internal 13 movement, especially in the lateral direction, is restricted by the matrix to filler bonding. 14 Therefore, during the compression stroke, the sample seems to be suppressed in a uni15 direction. The higher strain excitation might also cause relative displacement between the 16 sample and jaw at the contact surface resulting in a loss of stress [31].

17 The other quantities representing the viscoelastic properties of the MREs, i.e. field-dependent 18 modulus, loss tangent and MR effect, are determined from the stress-strain relationship. 19 Figures 11(a) and 11(b), respectively, show the storage modulus and loss modulus for all samples under different current intensities. Both the storage modulus and loss modulus have

21 the same trends. Taking the MRE $40 \mathrm{wt} \%$ data, the storage modulus rises according to the 22 increment in flux density, which is represented by the applied current. The increment is 23 limited to a particular current, and then it tends to level off. The same situation occurs for the 24 loss modulus of the sample. However, the lower fraction of MRE shows a slight increment in 
1 both the storage modulus and loss modulus. A relatively small difference between the off

2 state and maximum applied current can be influenced by certain factors, such as the 3 magnetite powder content, the powder dispersion (random or align), and the high initial 4 modulus of the base matrix. The loss tangent data are presented in Figure 11(c). The quantity 5 of loss tangent for carbon black filled rubber can exceed 0.8. This quantity is usually 6 possessed by hard rubber that has a high damping capability. The magnetic field effects on 7 the waste rubber based MRE influence the quantity of loss tangent. The increase in magnetic 8 field intensity makes the loss tangent grow gradually. Meanwhile, at the same flux density, 9 the loss tangent increases as the increment of magnetite weight fraction. The change in loss 10 tangent means that the damping property of each sample can be controlled by regulating a 11 proper magnetic flux density.

12 The MR effect is always the reference for the performance assessment of MREs. The 13 alteration in the ability of intrinsic properties can be directly evaluated from the value of the 14 MR effect. From this parameter, the reader can imagine how far the MRE is responding to the 15 appearance of the magnetic field. Figure 11(d) displays the MR effect of all samples for the 16 various applied currents. The presented data are calculated from the storage modulus values. 17 The highest MR effect is achieved by the MRE $40 \mathrm{wt} \%$, which is about $15 \%$, while the 18 lowest one is about 9\%. The MR effect of isotropic MREs is usually small. Therefore, most 19 researchers recommend the anisotropic type [32]. The viscoelastic properties of waste based 20 MREs are also investigated in terms of swept frequency and strain amplitude. The modulus 21 and loss tangent dependence on the magnetic field are presented in logarithmic scale. The 22 change in properties between a free magnetic field and under a magnetic field is shown by the 23 sample test at 0 and 5 A. Figure 12(a) demonstrates the rheological properties that cover 24 frequency ranges from 1 to $16 \mathrm{~Hz}$. The storage moduli of all MRE types increase gradually 25 with the increase in frequency. The consistent increment is also found in the magnetic field 26 variation. The growth of storage modulus is logarithmically linear. The prediction of the 27 storage modulus of the particular specimen at various frequencies (within the test span) can 28 refer to the graph. This is the benefit of using a logarithmic scale [33]. 


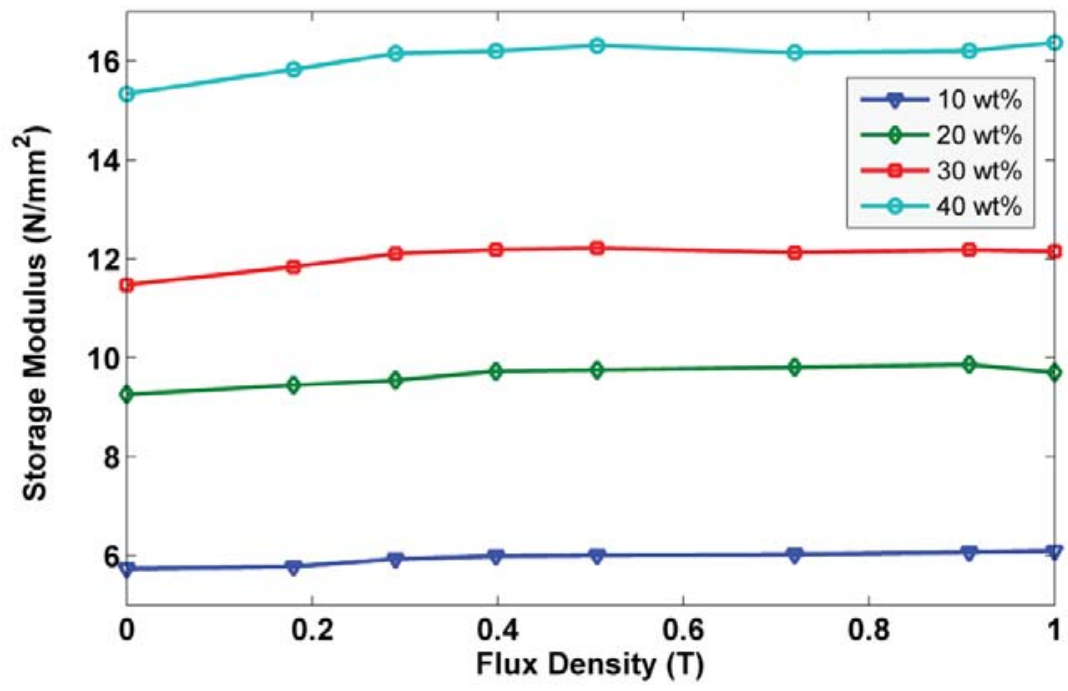

(a)

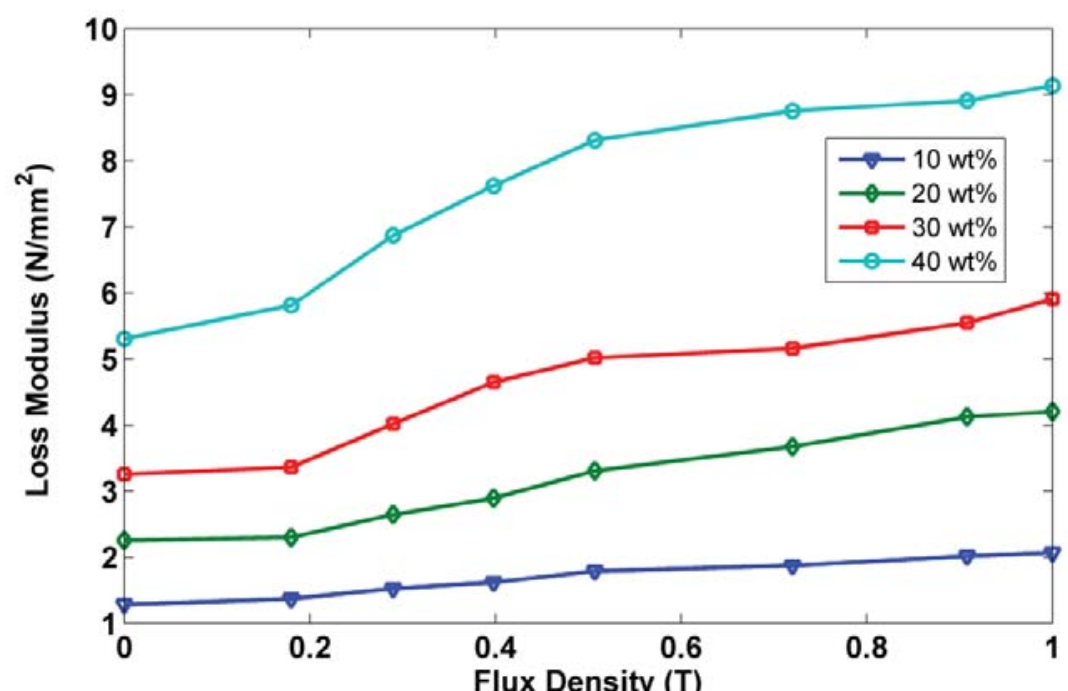

3

(b)

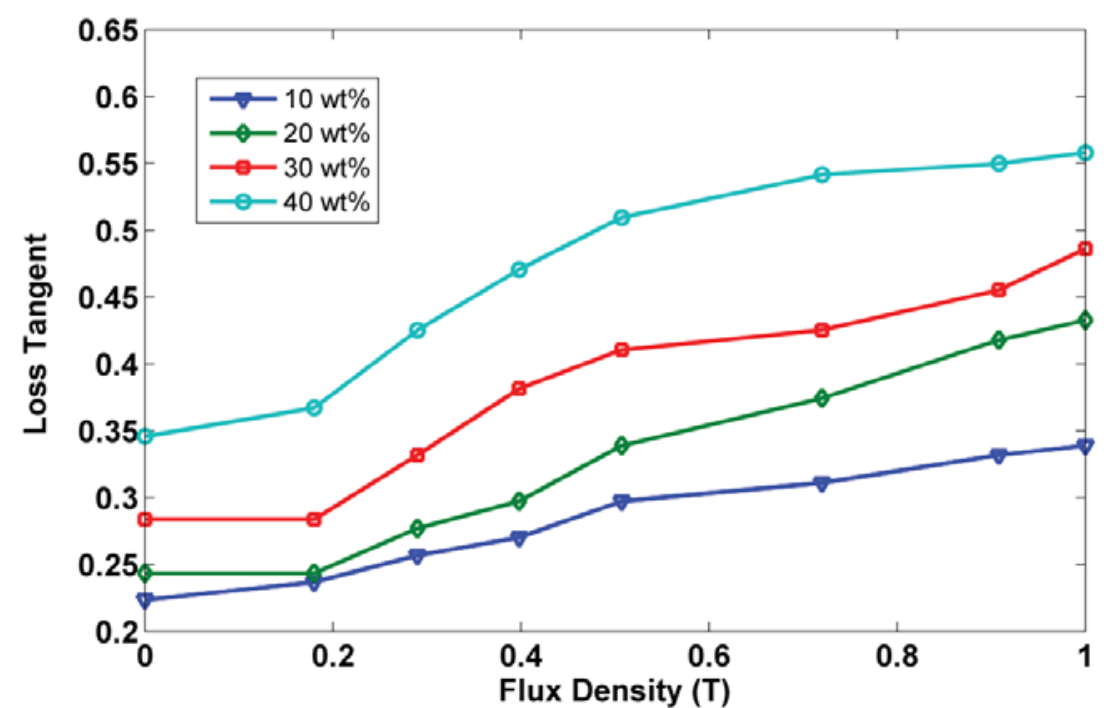




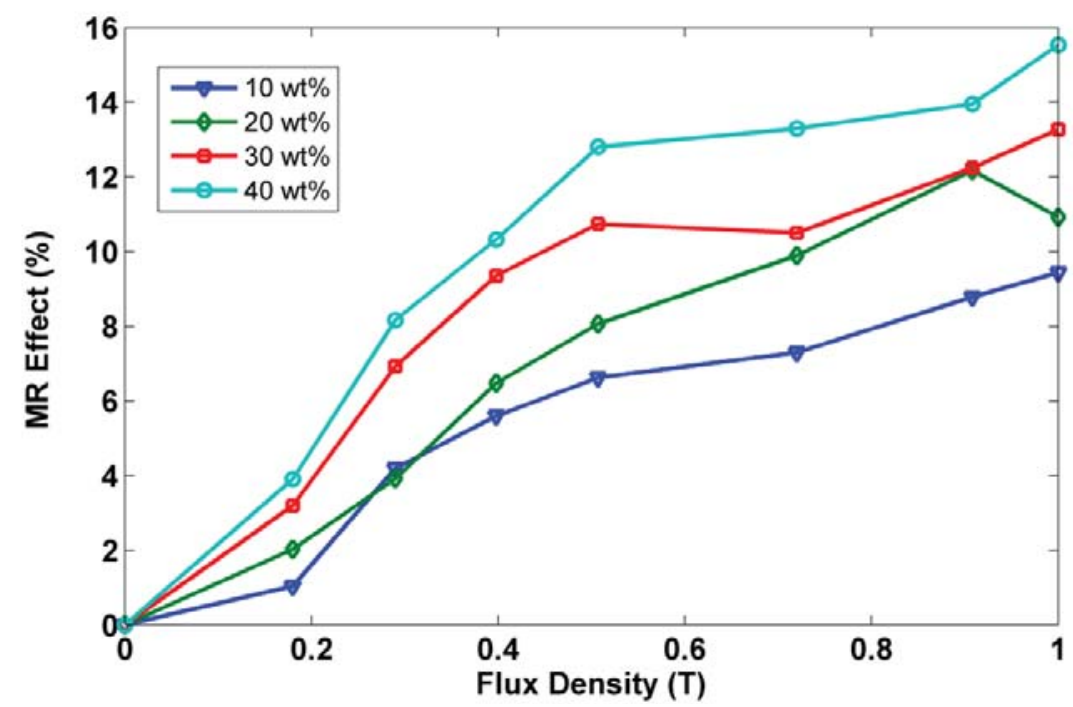

Figure 11. Viscoelastic behaviors of various samples of $1 \mathrm{~A}, 1 \mathrm{~Hz}$, and $10 \%$ amplitude: (a) storage modulus, (b) loss modulus, (c) loss tangent, and (d) MR effect

5 In contrast, the loss modulus and loss tangent show a different trend to the storage modulus in

6 the frequency domain. As can be seen in Figures 12(b) and (c), both the loss modulus and

7 loss tangent diminish gradually until the excitation frequency reaches $13 \mathrm{~Hz}$ and increases

8 later. At this frequency the storage modulus also increases following a reduction in value 9 above $13 \mathrm{~Hz}$. However, the values of the demagnetized condition are consistently higher than 10 that of the non-magnetized treatment. The slope in the storage modulus increment is lower 11 than that of the loss modulus and loss tangent. Based on this fact, it can be inferred that the 12 change in frequency is the dominant influence on the loss modulus and loss tangent. The 13 dissipated energy, which strongly correlates with the loss modulus, is more prominent than 14 the stiffness factor. 


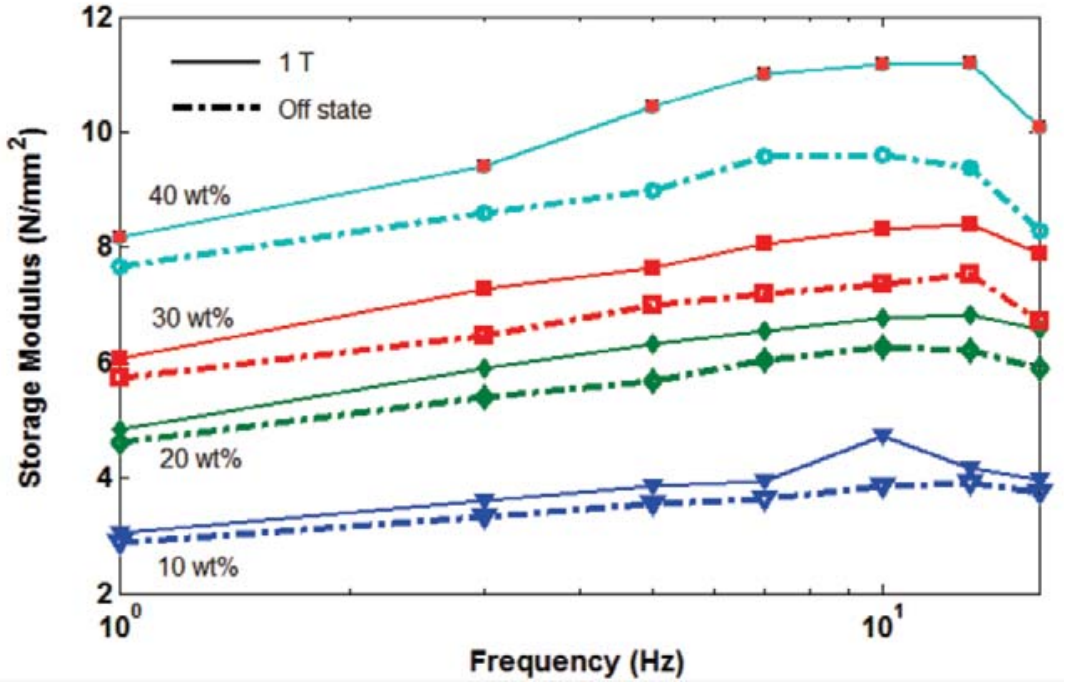

(a)

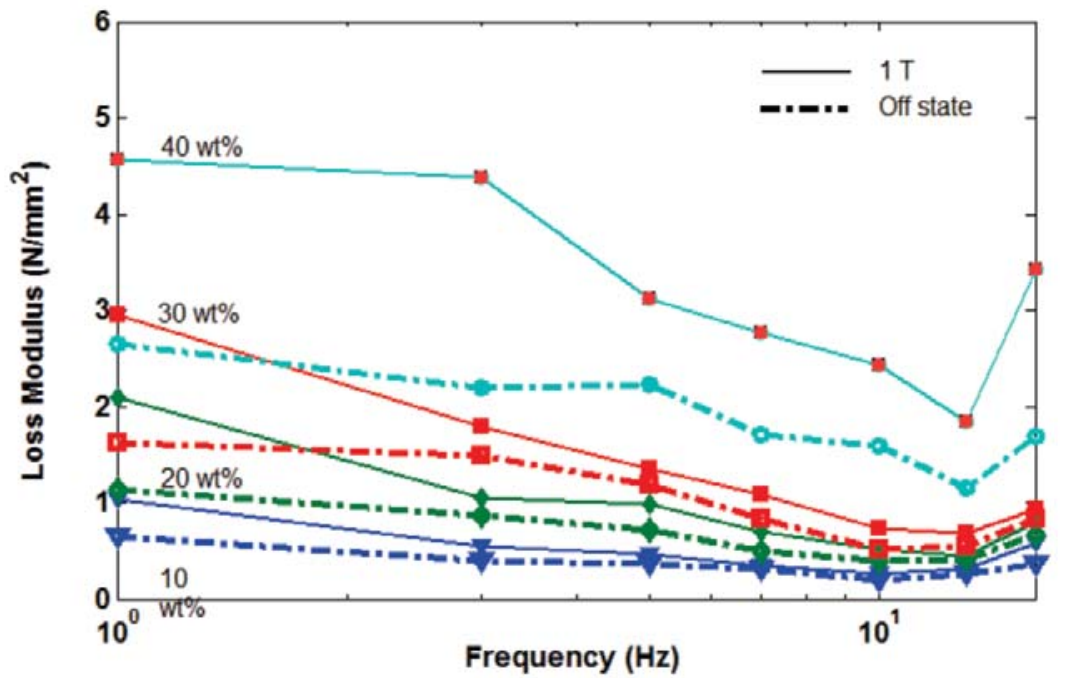

4

(b)

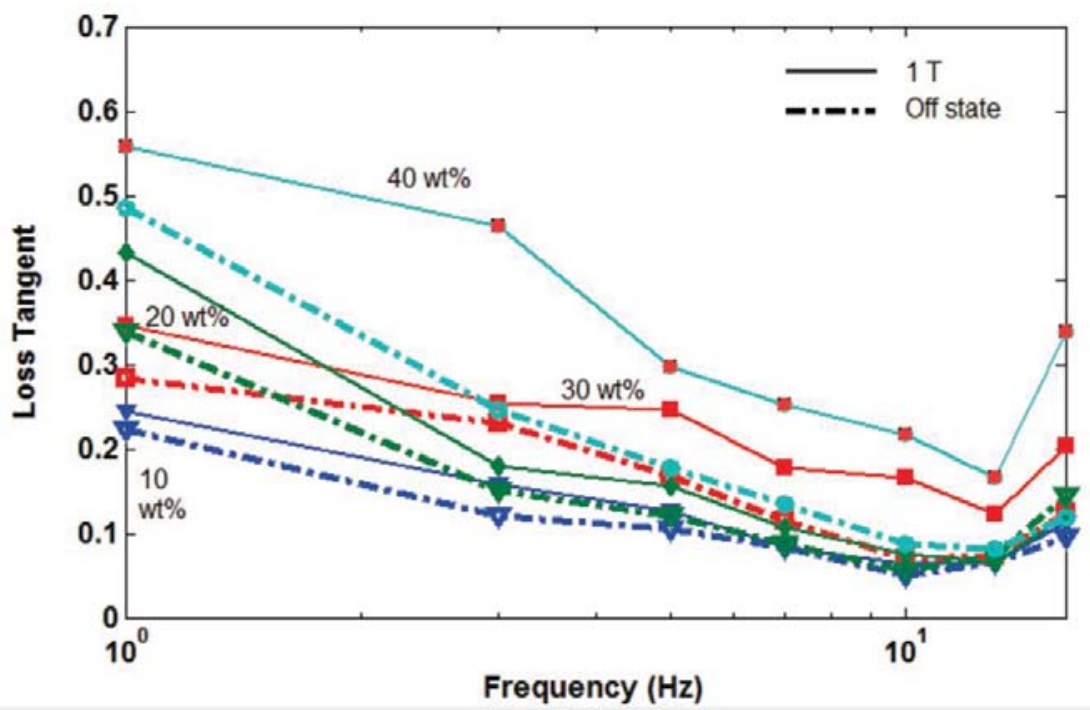


(c)

2 Figure 12. Viscoelastic behavior in swept frequency at off state and $1 \mathrm{~T}, 10 \%$ strain: (a) storage modulus, (b) loss modulus, and (c) loss tangent

4 The behaviors of waste based MREs at various compression strains are depicted in Figures 5 13(a) to (c). Figure 13(a) shows that the storage modulus drops steadily with a constant 6 excitation frequency of $1 \mathrm{~Hz}$. The decrease in storage modulus could be caused by the 7 microstructural deformation of the specimen. The high strain in compression mode 8 potentially destroys the bonding between the matrix and magnetizable powder. Therefore, the 9 stiffness of the MREs diminishes gradually as the result of microscopic bonding. The slope of 10 the decrement is most evident in the MRE $40 \mathrm{wt} \%$. The higher powder content reduces the 11 distance between particles. Hence, the slope of the lower filler content is flatter than for the 12 higher one. Different to the storage modulus behavior, the loss modulus and loss storage have 13 an incremental tendency after the drop in value in the first stage strain amplitude (1 to $3 \%$ 14 amplitude). This fact can be clearly seen in Figures 13(b) and (c). The off-state and on-state 15 conditions are consistently showing differences as per the previous phenomena. The closer 16 distance or lower thickness of the MREs is accompanied by higher magnetic field intensity, 17 as can be conceived from the finite element magnetic simulation undertaken in this work. The 18 current circumstance implies that the loss modulus and loss tangent strongly depend on the 19 increment in the strain amplitude. In general, the alteration in the storage and loss modulus, 20 and loss tangent under various magnetic fields, frequency and amplitude excitations clearly 21 confirms the control capability of the damping characteristics of the MREs proposed in this work.

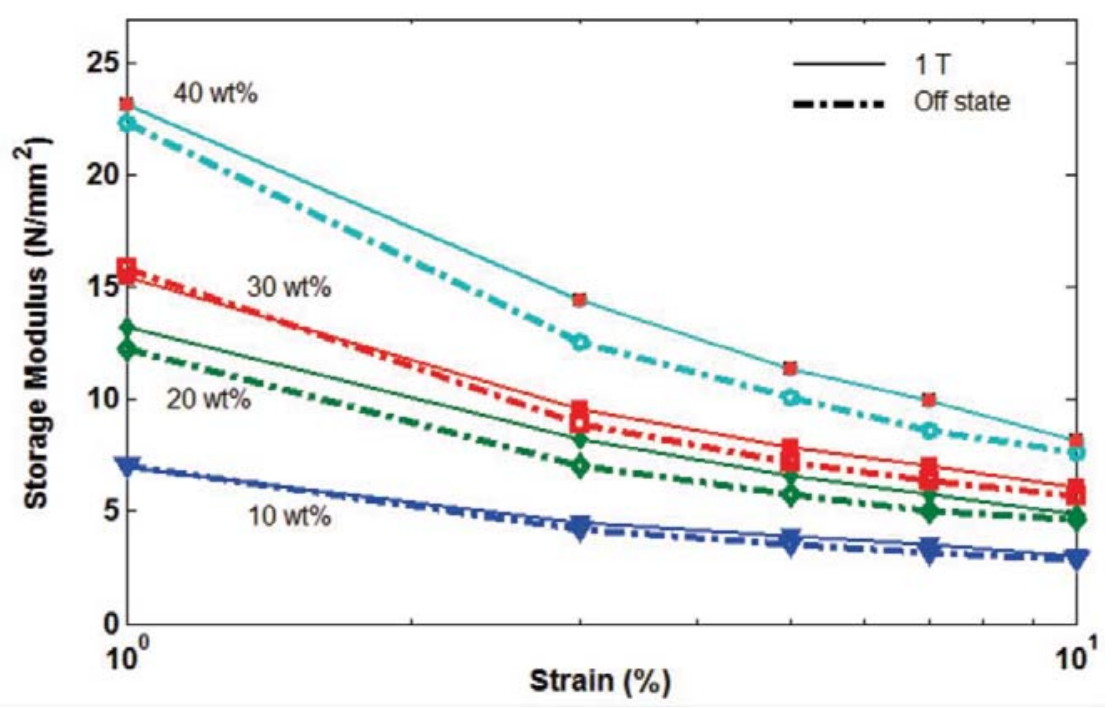




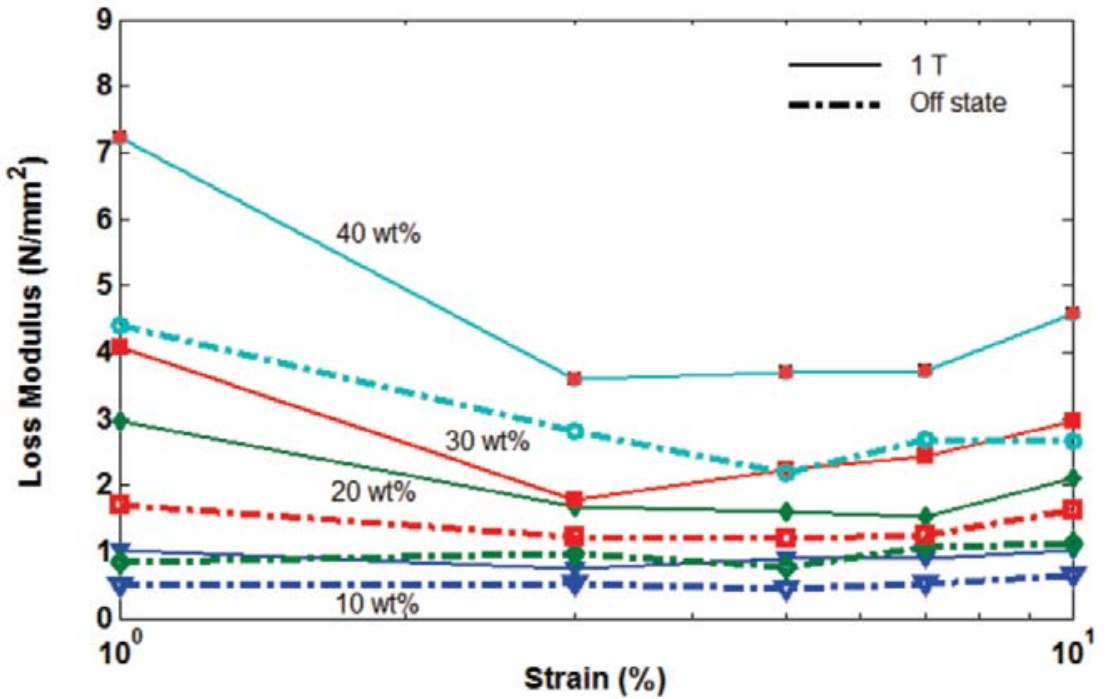

(b)

\section{4.6. Remarks}

8 The high MR effect is commonly preferred by researchers working in the MR materials area.

9 It is almost reached in the elastomeric base matrix with small viscoelastic properties under

10 zero magnetic fields, for example, soft elastomeric matrix. The soft elastomers are easily

11 found in miscible polymer based elastomers, such as silicone room temperature vulcanization

12 (RTV) and polydimethylsiloxane (PDMS). Therefore, most of the previous works on the

13 synthesis of MREs chose silicone RTV or another liquid-based elastomer as the matrix

14 carrier rather than synthetic and natural rubbers [4]. The low off-state property of MREs is 
1 indeed applicable to wide semi-active devices or systems. However, some applications need 2 relatively high off-state properties; for instance, seismic bearing load [34]. The rubber 3 bearing applied in the seismic application requires high damping and toughness for extended 4 span utilization. According to the application, the MR effect of the MREs is somehow 5 sacrificed [6]. Therefore, achieving a high MR effect in high mechanical properties (in zero 6 magnetic fields) of the MREs requires a major effort.

7 The synthesized MREs in this research are as previously described. In general, waste based 8 MREs have a high off-state modulus. The main reason being that the raw material of the base 9 matrix contains un-negligible carbon black. Thus, the modulus of pure reclaimed rubber is 10 much higher than for the conventionally used matrix in MREs. Furthermore, the insertion of 11 magnetite powder also affects the base modulus of the material. Therefore, it is 12 understandable that the maximum achievable MR effect is only about $15 \%$. However, this 13 new class of MRE provides unique properties, especially in the decrease of the storage 14 modulus in the scanned strain amplitude.

15 The reduction in the modulus in increasing strain is absent from the behavior of "pure gum" 16 rubber [35]. The phenomenon occurs when an active filler is inserted throughout the rubber;

17 for instance, carbon black, silica or metallic particles. The strain dependence modulus of 18 rubber reinforced particles has been formerly investigated by Payne [35,36]. This situation is 19 valid for all modes - compression, shear or tensile. The waste-based MREs show the effect 20 identified by Payne, as depicted in Figure 13(a), in which their storage modulus gradually 21 diminishes according to the increment in strain. The Payne effect can be an indicator of the 22 bonding strength between the particles and the matrix [37].

23 Another interesting behavior of the waste based MREs is their high-value loss tangent, as 24 depicted in Figure 11(c), in which the increment of loss tangent occurs when the current is 25 elevated. The loss tangent plays a significant role in viscoelastic materials, especially in 26 damping properties. The damping property of a regular particle reinforced elastomeric 27 composite is naturally influenced by the filler condition. The damping mechanism of this 28 kind of composite is attributed to certain factors, such as intrinsic damping, interparticle 29 friction, particle to matrix interaction and thermal-dislocation, caused by the applied strain 30 [38]. In MREs, the influencing factors include the effect of magneto-mechanical interaction. 31 So far, the investigation on damping mechanisms within the MREs lies in the model 32 predictions compared to the experimental result. The direct experimental investigation on the 33 particular factor affecting the damping mechanism has not yet been reported. Thus, the 
1 portion contributed by the influencing factors to the MRE damping has not been explicitly 2 quantified. However, most researchers state that the damping in MREs is dominantly 3 influenced by the particle-matrix interaction.

4 The Payne effect and loss tangent have a strong relationship. According to the viscoelastic 5 behavior of the waste based MREs, the microstructure change of MREs is affected by the 6 deformation, which will cause breakage and the recovery of a weak bonding. In this case, the 7 magnetite powder and waste rubber matrix form a small structure. The reclaimed rubber 8 interconnects the adjacent particles. Consequently, when the strain amplitude is applied to the 9 MRE sample, the inter-particle distance also changes. In the case of compression, the loading 10 direction is parallel to the longitudinal axis of the sample. Meanwhile, the deformation of the 11 MRE tends to be in the lateral direction. The cyclic strain leads to the particles being 12 repeatedly restrained and returned. The microscopic movement may break the particle-matrix 13 bond, and, hence, the small structures are destroyed. This phenomenon may cause a decrease 14 in the storage modulus at the same time. Along with the cyclic loading and the existence of 15 the relative microscopic movement between the magnetite particles and rubber matrix, the 16 friction will increase, resulting in an increment in the loss tangent. According to the 17 microstructure results, as depicted in Figure 4, a major break in the particle-matrix bonding 18 has not been found. The photograph was taken from a sliced sample after testing. Thus, it is 19 predicted that the relative movement that occurred in the synthesized MREs must be of a subtle nature, and would appear after cyclic loading and long term excitation. Furthermore, 21 the waste rubber based MREs are believed to have a broad range of controllable damping 22 properties that are suitable for a wide arrange of applications, such as semi-active devices, 23 and systems, such as seismic absorbers.

24 However, it is acknowledged that the proposed MREs still have certain drawbacks. For 25 example, the proposed MREs need to be further improved to have better viscoelastic 26 properties. The potentially important issues that require exploration in future studies are the 27 matrix-filler compatibility and the filler structure within the matrix. The compatibility of the 28 waste rubber and magnetite can be improved through surface particle modification or an 29 additional bonding agent, as investigated in earlier studies [39-41]. The aligning of particles 30 within the MRE matrix is also expected to increase the MR effect. In addition, the 31 modification of the HTHP method is another challenging problem in respect of the 32 fabrication technique. 


\section{5. Reliability Issues}

2 The primary requirement for the implementation of reclaimed waste tire rubber is that the 3 rubber should have adequate durability and life span. The requirement should also be 4 appointed to the MRE based devices, such as adaptive tunable vibration absorbers, since they 5 are constructed of soft (elastomeric matrix) and hard materials (magnetizable powder). Most

6 of the devices are implemented in fluctuating loads, with environmental alteration in the long 7 term. The stability and degradation of the MREs must be the primary consideration in various 8 different operating conditions. Thermal analyses, such as differential scanning calorimetry 9 (DSC), thermogravimetric analysis (TGA/DTA), dynamic mechanical-thermal analysis 10 (DMTA), aging test and thermal oxidation, as well as UV oxidation are usually conducted to 11 investigate the performance of the elastomeric materials, including MREs, to determine their 12 response to thermal loads. Studies on the thermal analysis of MREs based on virgin 13 elastomers can be found in some previous works [32,42,43,43-47]. Since the specimens 14 studied in this report had waste rubber as the MRE matrix, there is no report that precisely

15 explains the stability and degradation. The broad investigation on the thermal analyses of the 16 waste rubber based MRE will be presented in a future report.

17 The approach for predicting the durability of the waste rubber based MREs can be based on 18 an initial assessment of the thermal stability of the reclaimed waste rubber. The investigation 19 on the thermal analyses of reclaimed waste rubber, especially ground tire rubber, has been 20 explored in some previous reports. The thermal analyses of reclaimed tire rubber waste, 21 without mixing with virgin rubber, has been investigated by some researchers [48-50]. In the 22 investigation, the method for compounding crumb rubber utilizes a screw mixer at a 23 temperature of $180{ }^{\circ} \mathrm{C}$, and an applied shear pressure of less than the pressure in the HPHT 24 reclamation process by Morin et al. [51], with additional vulcanization agents. In the work 25 performed by De et al. [52], the reclaimed tire rubber retains good tensile and hardness 26 properties. Even with the additional concentration of TMTD, the phenomenon showed the 27 anti-aging characteristics of the reclaimed rubber. The results confirmed that the aging 28 characteristics of reclaimed rubber were comparable to the virgin based natural rubber. 29 Formela et al. [49] investigated the thermomechanical reclaiming process of waste tire rubber 30 and concluded that the reclaiming process influenced the thermal characteristics; this relation 31 was confirmed by the Horikx theory. Meanwhile, Shi et al. [50] upheld the stability of 32 reclaimed waste tires through DSC examination. It was reported that the thermal glass

33 transition temperature was slightly different to that of raw natural rubber. Based on the 
1 aforementioned investigation, the reclaimed waste tire rubber has a reasonable durability near

2 to that of virgin rubber. Accordingly, this could be used as preliminary judgment for the

3 durability of waste tires based MREs albeit the prediction must be scientifically proven

4 through thermal analysis.

\section{6. Conclusion}

6 In this work, a new type of MRE based on the waste tire rubber was fabricated and the field-

7 dependent material characterizations were undertaken in both static and dynamic conditions.

8 Particularly, this work explored the possibility of waste tire rubber as the primary matrix of

9 magnetorheological elastomers. The reclaimed rubber based MREs were synthesized using a

10 proven technique, namely, high-temperature high-pressure sintering. Some physical-chemical

11 examinations were conducted to investigate the microstructural properties; magnetic

12 properties and thermal glass transition. The magnetite powder was dispersed randomly within

13 the rubber matrix without meaningful agglomeration. The thermal glass transition

14 temperature of the waste tire rubber based MRE was determined at $-60.6 \pm 0.5{ }^{\circ} \mathrm{C}$, which is

15 comparable with reclaimed waste tire rubber (without magnetite powder). The development

16 of an electromagnetic device for compression test was performed from the magnetic circuit

17 analysis, finite element simulation, and fabrication. The validity of the simulation results on

18 magnetic field generation were proven by comparing with the experimental results. Based on

19 this validated model, the flux density across the MREs was accurately predicted through the

20 simulation. The electromagnetic device was then integrated into the fatigue dynamic machine

21 for MRE compression tests. The produced MREs have shown their controllable properties

22 with the appearance of a magnetic field. The effect of increasing frequency on the MREs

23 caused a slight increment in the storage modulus and a decrement in the loss modulus and

24 loss tangent. Meanwhile, the growth of strain amplitude caused a meaningful decrement in

25 the storage modulus (Payne effect) and an increment in the loss modulus and loss tangent. In

26 addition, it was demonstrated that the reclaimed rubber based MREs possess a wider tunable

27 property of damping capacities than for the stiffness properties. Consequently, this class of

$28 \mathrm{MRE}$ is an excellent candidate for applications that require controllable high damping

29 capability, such as seismic shock absorbers or for the automotive industry. Future works

30 concerning the utilization of waste tire rubber, such as MRE matrix, should concentrate on

31 the reliability and durability issue that is normally conducted through thermal analyses as

32 well as the aging effect in respect of its rheological properties. 


\section{7. Acknowledgement}

2 This work has been supported by collaborative research between MJIIT-UTM (PRGS Grant

3 No.4L667 and RUG No.06H06) as well as Universitas Sebelas Maret (Mandatory Research

4 Grant 2015, No : 698 / UN27/ PN/2015).

\section{8. References}

6 [1] De D, Panda PK, Roy M, Bhunia S, Jaman AI. Reinforcing effect of nanosilica on the

[5] Kallio M, Lindroos T, Aalto S, Järvinen E, Kärnä T, Meinander T. Dynamic compression testing of a tunable spring element consisting of a magnetorheological elastomer. Smart Mater Struct 2007;16:506-14.

[6] Li R, Sun LZ. Viscoelastic Responses of Silicone-Rubber-Based Magnetorheological Elastomers Under Compressive and Shear Loadings. J Eng Mater Technol 2013;135:021008.

[7] Farshad M, Benine A. Magnetoactive elastomer composites. Polym Test 2004;23:347-53.

[8] Boczkowska A, Awietjan SF, Wroblewski R. Microstructure-property relationships of urethane magnetorheological elastomers. Smart Mater Struct 2007;16:1924-30.

[9] Gordaninejad F, Wang X, Mysore P. Behavior of thick magnetorheological elastomers. J Intell Mater Syst Struct 2012;23:1033-9.

[10] Sorrentino L, Aurilia M, Forte G, Iannace S. Composite Polymeric Foams Produced by Using Magnetic Field. Adv Sci Technol 2008;54:123-6.

[11] Gong QC, Wu JK, Gong XL, Fan YC, Xia HS. Smart polyurethane foam with magnetic field controlled modulus and anisotropic compression property. RSC Adv 2013;3:3241. 
1 [12] Bica I, Anitas EM, Bunoiu M, Vatzulik B, Juganaru I. Hybrid magnetorheological

4 [13] Zhu XL, Meng YG, Tian Y. Nonlinear pressure-dependent conductivity of magnetorheological elastomers. Smart Mater Struct 2010;19:117001.

[14] Liao G, Gong X, Xuan S. Magnetic field-induced compressive property of magnetorheological elastomer under high strain rate. Ind Eng Chem Res 2013;52:8445-53.

[15] Liao G, Gong X, Xuan S, Guo C, Zong L. Magnetic-field-induced normal force of magnetorheological elastomer under compression status. Ind Eng Chem Res 2012;51:3322-8.

[16] Koo JH, Khan F, Jang DD, Jung HJ. Dynamic characterization and modeling of magneto-rheological elastomers under compressive loadings. Smart Mater Struct 2010;19:117002.

[17] Song HJ, Wereley NM, Bell RC, Planinsek JL, Filer II JA. Field dependent response of magnetorheological elastomers utilizing spherical Fe particles versus Fe nanowires. J Phys Conf Ser 2009;149:012097.

[18] Bengawan Sumber Baru P. 2013 Technical assesment for ground tire rubber in PT. Bengawan Sumber Baru. Surakarta: 2014.

[19] Mitsumata T, Ohori S, Honda A, Kawai M. Magnetism and viscoelasticity of magnetic elastomers with wide range modulation of dynamic modulus. Soft Matter 2013;9:904.

[20] Bergstrom JS, Boyce MC. Constitutive modeling of the large strain time-dependent behavior of elastomer. J Mech Phys Solid 1998;46:931-54.

[21] Zhang CX, Bowler N, Lo C. Magnetic characterization of surface-hardened steel. J Magn Magn Mater 2009;321:3878-87.

[22] Goodwin JW, Hughes RW. Rheology for chemist an introduction. Cambridge: RSC Publishing; 2008.

[23] Mitsumata T, Ohori S. Magnetic polyurethane elastomers with wide range modulation of elasticity. Polym Chem 2011;2:1063.

[24] Mitsumata T, Ohori S, Chiba N, Kawai M. Enhancement of magnetoelastic behavior of bimodal magnetic elastomers by stress transfer via nonmagnetic particles. Soft Matter 2013;9:10108.

[25] Neelakanta PS. Handbook of electromagnetic materials. CRC Press LLC; 1995. 
1 [26] Kong I, Hj Ahmad S, Hj Abdullah M, Hui D, Yusoff AN, Puryanti D. Magnetic and microwave absorbing properties of magnetite thermoplastic natural rubber nanocomposites. J Magn Magn Mater 2010;322:3401-9.

[27] Ahmed S, Abdullah I, Abdullah M, Wai WC. Electrical and magnetic properties of barium ferrite thermoplastic natural rubber (TPNR) composites. Sci Int 1998;16:3757.

[28] Kim KD, Pernecker T, Saddow T. SBR latex polymers with improved auto-adhesion. Rubber Plast News 2012:15-8.

[29] Guth E. Theory of Filler Reinforcement. J Appl Phys 1945;16:20.

[30] Koo JH, Khan F, Jang DD, Jung HJ. Dynamic characterization and modeling of magneto-rheological elastomers under compressive loadings. J Phys Conf Ser 2010;149:012093.

[31] Zhu JT, Xu ZD, Guo YQ. Magnetoviscoelasticity parametric model of an MR elastomer vibration mitigation device. Smart Mater Struct 2012;21:075034.

[32] Zhang W, Gong XL, Jiang WQ, Fan YC. Investigation of the durability of anisotropic magnetorheological elastomers based on mixed rubber. Smart Mater Struct 2010;19:085008.

[33] Tian TF, Li WH, Alici G, Du H, Deng YM. Microstructure and magnetorheology of graphite-based MR elastomers. Rheol Acta 2011;50:825-36.

[34] Li YC, Li JC, Li WH, Samali B. Development and characterization of a magnetorheological elastomer based adaptive seismic isolator. Smart Mater Struct 2013;22:035005.

[35] Payne A. The Dynamic Properties of Carbon Black-Loaded Natural Rubber Vulcanizates. Part I. J Appl Polym Sci 1962;VI:57-63.

[36] Payne A. The Dynamic Properties of Carbon Black Loaded Natural Rubber Vulcanizates. Part II. J Appl Polym Sci 1962;VI:368-72.

[37] Li JF, Gong XL, Zhu H, Jiang WQ. Influence of particle coating on dynamic mechanical behaviors of magnetorheological elastomers. Polym Test 2009;28:331-7.

[38] Yang J, Gong XL, Deng HX, Qin LJ, Xuan SH. Investigation on the mechanism of damping behavior of magnetorheological elastomers. Smart Mater Struct 2012;21:125015.

[39] Pickering KL, Raa Khimi S, Ilanko S. The effect of silane coupling agent on iron sand for use in magnetorheological elastomers Part 1: Surface chemical modification and characterization. Compos Part A Appl Sci Manuf 2015;68:377-86. 
1 [40] Behrooz M, Sutrisno J, Zhang LY, Fuchs A, Gordaninejad F. Behavior of magnetorheological elastomers with coated particles. Smart Mater Struct 2015;24:035026.

4 [41] Fuchs A, Sutrisno J, Gordaninejad F, Caglar MB, Yanming L. Surface polymerization of iron particles for magnetorheological elastomers. J Appl Polym Sci 2010;117:93442.

[42] Gong XL, Chen L, Li JF. Study of Utilizable Magnetorheological Elastomers. Int J

[44] Xu YG, Gong XL, Xuan SH, Li XF, Qin LJ, Jiang WQ. Creep and recovery behaviors of magnetorheological plastomer and its magnetic-dependent properties. Soft Matter 2012;8:8483.

[45] Ivaneyko D, Toshchevikov V, Saphiannikova M, Heinrich G. Magnetorheological Elastomers. Encycl Polym Nanomater 2014:1-8.

[46] Zhang W, Gong XL, Xuan SH, Jiang WQ. Temperature-Dependent Mechanical Properties and Model of Magnetorheological Elastomers. Ind Eng Chem Res 2011;50:6704-12.

[47] Wu JK, Gong XL, Chen L, Xia HS, Hu ZG. Preparation and Characterization of Isotropic Polyurethane Magnetorheological Elastomer Through In Situ Polymerization. J Appl Polym Sci 2009;114:901-10.

[48] De D, Das A, Dey B, Debnath SC, Roy BC. Reclaiming of ground rubber tire (GRT) by a novel reclaiming agent. Eur Polym J 2006;42:917-27.

[49] Formela K, Cysewska M, Haponiuk J. The influence of screw configuration and screw speed of co-rotating twin screw extruder on the properties of products obtained by thermomechanical reclaiming of ground tire rubber. Polimery 2014;59:170-7.

[50] Shi JW, Zou H, Ding LL, Li XL, Jiang K, Chen T, et al. Continuous production of liquid reclaimed rubber from ground tire rubber and its application as reactive polymeric plasticizer. Polym Degrad Stab 2014;99:166-75.

[51] Morin JE, Williams DE, Farris RJ. A Novel Method to Recycle Scrap Tires: HighPressure High-Temperature Sintering. Rubber Chem Technol 2002;75:955-68.

[52] De D, Das A, De D, Dey B, Debnath SC, Roy BC. Reclaiming of ground rubber tire (GRT) by a novel reclaiming agent. Eur Polym J 2006;42:917-27. 
